\title{
Cardiovascular disease detection using machine learning and carotid/ femoral arterial imaging frameworks in rheumatoid arthritis patients
}

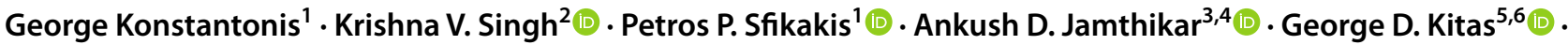

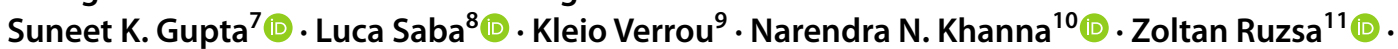

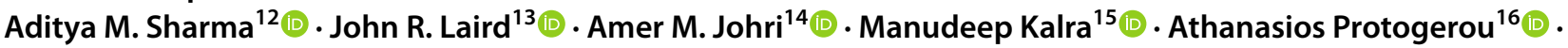 \\ Jasjit S. Suri ${ }^{17}$
}

Received: 25 October 2021 / Accepted: 29 November 2021

(c) The Author(s), under exclusive licence to Springer-Verlag GmbH Germany, part of Springer Nature 2021

\begin{abstract}
The study proposes a novel machine learning (ML) paradigm for cardiovascular disease (CVD) detection in individuals at medium to high cardiovascular risk using data from a Greek cohort of 542 individuals with rheumatoid arthritis, or diabetes mellitus, and/or arterial hypertension, using conventional or office-based, laboratory-based blood biomarkers and carotid/ femoral ultrasound image-based phenotypes. Two kinds of data (CVD risk factors and presence of CVD—defined as stroke, or myocardial infarction, or coronary artery syndrome, or peripheral artery disease, or coronary heart disease) as ground truth, were collected at two-time points: (i) at visit 1 and (ii) at visit 2 after 3 years. The CVD risk factors were divided into three clusters (conventional or office-based, laboratory-based blood biomarkers, carotid ultrasound image-based phenotypes) to study their effect on the ML classifiers. Three kinds of ML classifiers (Random Forest, Support Vector Machine, and Linear Discriminant Analysis) were applied in a two-fold cross-validation framework using the data augmented by synthetic minority over-sampling technique (SMOTE) strategy. The performance of the ML classifiers was recorded. In this cohort with overall 46 CVD risk factors (covariates) implemented in an online cardiovascular framework, that requires calculation time less than $1 \mathrm{~s}$ per patient, a mean accuracy and area-under-the-curve (AUC) of $98.40 \%$ and $0.98(p<0.0001)$ for CVD presence detection at visit 1 , and $98.39 \%$ and $0.98(p<0.0001)$ at visit 2 , respectively. The performance of the cardiovascular framework was significantly better than the classical CVD risk score. The ML paradigm proved to be powerful for CVD prediction in individuals at medium to high cardiovascular risk.
\end{abstract}

Keywords Cardiovascular risk estimation · Cardiovascular disease · Three-year follow-up · Conventional risk factors . Ultrasound · And machine learning

\begin{tabular}{|c|c|c|c|}
\hline \multicolumn{2}{|c|}{ Abbreviations } & \multirow[t]{3}{*}{ Cluster 3} & \multirow{3}{*}{$\begin{array}{l}\text { Fusion of office-based biomarker, labora } \\
\text { tory-based biomarker, and carotid ultra- }\end{array}$} \\
\hline ANOVA & Analysis of variance & & \\
\hline ASCVD & Atherosclerotic cardiovascular disease & & \\
\hline AUC & Area-under-the-curve & CUSIP & Carotid ultrasound image phenotype \\
\hline BMI & Body mass index & $\mathrm{CV}$ & Cross-validation \\
\hline CAD & Coronary artery disease & CVD & Cardiovascular disease \\
\hline CCVRC & $\begin{array}{l}\text { Conventional cardiovascular risk } \\
\text { calculators }\end{array}$ & CVD-3YFU & $\begin{array}{l}\text { Cardiovascular disease risk-three-year } \\
\text { follow-up }\end{array}$ \\
\hline Cluster 1 & Conventional office-based biomarkers & CVD-CR & Cardiovascular disease-current risk \\
\hline \multirow[t]{4}{*}{ Cluster 2} & Fusion of office-based biomarker and & CVE & Cardiovascular events \\
\hline & laboratory-based biomarkers & DM & Diabetes mellitus \\
\hline & & $\mathrm{FH}$ & Family history \\
\hline & & FNR & False-negative rate \\
\hline \multirow{3}{*}{\multicolumn{2}{|c|}{$\begin{array}{l}\text { Jasjit S. Suri } \\
\text { jasjit.suri@ atheropoint.com }\end{array}$}} & FPR & False-positive rate \\
\hline & & FRS & Framingham risk score \\
\hline & & HTN & Hypertension \\
\hline
\end{tabular}




$\begin{array}{ll}\text { IPN } & \text { Intraplaque neovascularization } \\ \text { LBBM } & \text { Laboratory-based biomarker } \\ \text { LDA } & \text { Linear discriminant analysis } \\ \text { ML } & \text { Machine learning } \\ \text { MPH } & \text { Maximum plaque height } \\ \text { NPV } & \text { Negative predictive value } \\ \text { OBBM } & \text { Office-based biomarker } \\ \text { PE } & \text { Performance evaluation matrices } \\ \text { PPV } & \text { Positive predictive value } \\ \text { RA } & \text { Rheumatoid arthritis } \\ \text { RF } & \text { Random forest } \\ \text { ROC } & \text { Receiver operating-characteristics } \\ \text { RRS } & \text { Reynolds risk score } \\ \text { SCORE } & \text { Systematic coronary risk evaluation } \\ \text { SMOTE } & \text { Synthetic minority over-sampling technique } \\ \text { SMOTE 5X } & \text { Five times synthetic minority } \\ & \text { over-sampling } \\ \text { SVMrbf } & \text { Support vector machine with the radial } \\ & \text { basis function } \\ \text { TPA } & \text { Total plaque area } \\ \text { TRF } & \text { Traditional risk factors } \\ \text { WHO } & \text { World Health Organization } \\ \end{array}$

\section{Introduction}

Individuals with rheumatoid arthritis (RA), diabetes mellitus (DM), and arterial hypertension are at increased cardiovascular disease (CVD) risk due to the accumulation of disease-associated or conventional CVD risk factors (e.g., obesity, dyslipidemia) [1,2]. The patients with RA have a two- to three-fold increased risk of cardiovascular events (CVE) compared to the normal population [3, 4]. Further, RA and CVD share common risk factors such as gender, hyperlipidemia, hypertension, diabetes, body mass index, physical inactivity, and smoking, known as traditional risk factors (TRF). Because of this fact, most of the conventional cardiovascular risk calculators (CCVRC) have been adopted to predict the CVD risk in RA patients.

CCVRC are used to predict the CVD risk in non-RA and RA patients, however they often either under-estimate or over-estimate the CVD risk in patients [5-7]. This is mainly due to their dependence on TRF, which does not fully explain the increased CVD risk, especially in RA [3, 8-11]. Subclinical atherosclerosis is a common early phenomenon in both RA and non-RA individuals, described by the growth of atherosclerotic plaque [12], and can be used in CVD risk stratification beyond TRF.

The use of non-invasive and economical imaging modalities such as carotid and femoral ultrasound can capture the growth of atherosclerotic plaque. Two popular carotid ultrasound image-based phenotypes (CUSIP) such as carotid intima-media thickness and carotid plaque area are considered as the surrogate indicator of coronary artery disease (CAD) [13-20]. For example, RA patients reportedly have higher values of this CUSIP compared to non-RA patients and, therefore, these atherosclerotic plaque-based phenotypes could be used for an accurate CVD risk assessment [21-24]. There are two major drawbacks with CCVRC (i) they do not integrate CUSIP in their risk prediction model and (ii) they do not handle the complex nonlinear association between several CVD risk factors and the CVE endpoints [12]. Lastly, since CCVRC is regression-based, they have limited capability to handle a large number of risk predictors.

ML-based systems have reported better and promising risk assessment compared to existing CCVRC in non-RA studies [12, 25]. We, therefore, hypothesize, that the MLbased systems can also provide better CVD risk assessment in RA/non-RA patients compared to CCVRC. We further hypothesize that this relation holds when TRF is fused with CUSIP in the ML framework instead of using TRF alone. The objective of the proposed study is to perform ML-based CVD risk stratification (so-called "ML-effect") of both RA and non-RA patients while handling the class imbalance using synthetic minority over-sampling technique (SMOTE), referred as "SMOTE-effect", between binary classes (such as CVD and No-CVD). Furthermore, this study examined the performance of ML-based systems at two distinct time points (i) visit 1 and (ii) visit 2 (after 3-year, against three popular CCVRC (such as FRS, ASCVD, and SCORE), referred to as the "Follow-up effect". Further, for lower cost reasons, especially in low-income countries, it is important to provide CVD risk assessment using the TRF. Therefore, another objective of the proposed study is to investigate the performance of ML-based CVD risk assessment systems considering (i) office-based risk biomarkers (OBBM) alone, (ii) using the combination of OBBM and laboratory-based biomarkers (LBBM), and (iii) and further using the combination of OBBM, LBBM, and CUSIP (referred as "Cluster-effect"). Figure 1 shows the global architecture of the proposed online ML-based CVD risk stratification system. The CVD risk assessment on a test patient is predicted by fusing OBBM, LBBM, CUSIP, and transforming these by the offline training ML-based model. The test-classifier can be the same as the online classifier. The predicted CVD risk is depicted by the binary colour code (red-CVD or greenNo CVD).

The four main innovations of this study are as follows: (i) Cluster Effect: For the first time, three diverse types of risk predictors such as OBBM, LBBM, and CUSIP are used under the ML framework to predict the CVD risk in RA participants. (ii) SMOTE Effect: To handle the problem of unequal classes (also categorically called class-imbalance), this study also shows the performance of an ML-based system with an imbalanced and balance dataset using the SMOTE 


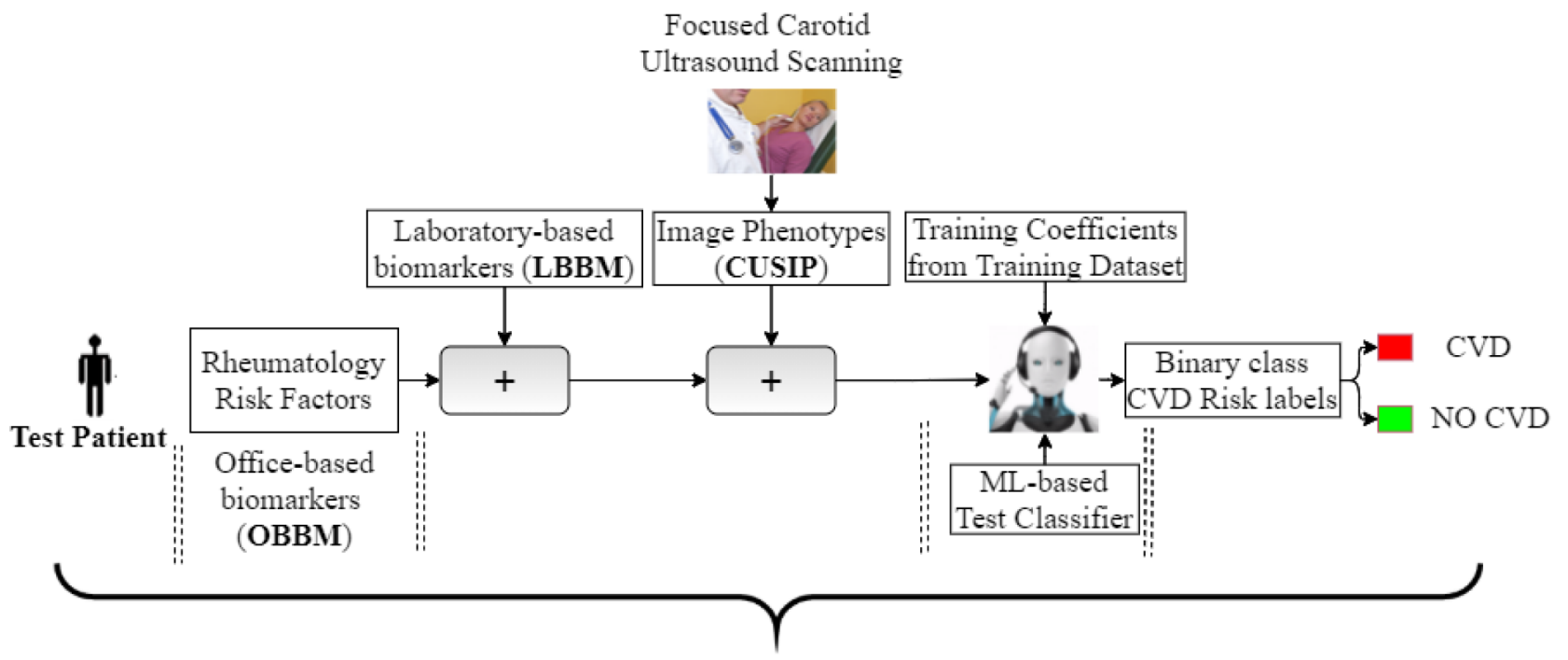

Global Architecture

Fig. 1 The global architecture of the proposed ML-based CVD risk stratification system. CVD cardiovascular disease, $M L$ machine learning

algorithm. (iii) Follow-up Effect: This is the first study that provided ML-based CVD risk stratification for RA patients at two distinct time points (visit 1: baseline (CVD-CR) and visit 2: 3-year follow-up (CVD-3YFU). Three popular ML-based algorithms (random forest (RF), support vector machine (SVM), and linear discriminant analysis (LDA)) are used to investigate the CVD risk presence of participants with and without RA. (iv) ML Effect: the performance of the ML-based CVD risk assessment system is benchmarked against three popular CCVRC (FRS, SCORE, and ASCVD).

\section{Methodology}

\section{Patient demographics}

This is a single-center cross-sectional design study at two different time points (visit 1 and visit 2). Consecutive consenting to participate individuals that were examined at the outpatient rheumatology, diabetes and hypertension clinics of the "LAIKO" general hospital in Athens Greece were recruited. All recruited participants were examined at that cardiovascular laboratory of our department, to have ultrasound tests and to optimize their cardiovascular risk stratification. This study used a total of 542 Greek European patients, out of which—at visit 1: baseline-535 individuals patients were free of established CVD and 7 patients had CVD (CVD-CR), such as stroke, or myocardial infarction, or coronary artery syndrome, or peripheral artery disease, or coronary heart disease, or death, collectively referred as CVE. At visit 2, after a 3-year follow-up (3YFU), out of the 542 patients, 8 patients had established CVD. The mean age of the study participants with CVD-CR was $53.07 \pm 13.5$ years (ranging between 15 and 90 years), mean weight was $77.27 \pm 17.0 \mathrm{Kgs}, 37.5 \%$ had hypertension, $76 \%$ were smokers, and $27.1 \%$ had hyperlipidemia.

\section{Ultrasound image acquisition}

Carotid and femoral atherosclerotic plaque burden is a surrogate marker for CVE, which is increased in individuals with high CVD risk [24, 26-29]. The carotid and femoral ultrasound examination in all study participants were performed using GE Vivid (Vivid E9 Ultrasound System, GE Healthcare), equipped with a linear probe-type M12L (5.6-14 MHz), as presented in our previous study [30]. The American Society of Echocardiography (ASE) guidelines were used for the ultrasound image acquisition. A dedicated and very experienced (more than 4000 patients examined) trained technician performed all the measurements. CUSIP measurements were recorded from ultrasound scans [30, 31]. The American Society of Echocardiography (ASE) Task Force [32] recommendations were used for image acquisition. The patient was made to lie in the supine position with the head tilted backwards for examining the carotid arteries. Two-step standardized image acquisition protocol was adapted (i) carotid arteries were identified in a transverse position orthogonal to the blood flow, and (ii) probe was titled by $90^{\circ}$ to acquire the far wall of the longitudinal scans (parallel to the blood flow) of the carotid arteries. The above procedure was used and discussed in our previous studies [33-36]. 


\section{Hypothesis}

We hypothesize that (i) Cluster effect: the effect of CUSIP as a biomarker in ML framework can improve the CVD detection in RA and non-RA participants and we believe that the accuracy should follow the pattern $(\mathrm{OBBM}+\mathrm{LBBM}+\mathrm{CUSI}$ P) $>(\mathrm{OBBM}+\mathrm{LBBM})>$ OBBM . (ii) Follow-up effect: using ML, we were able to predict CVD-3YFU, and we believe that the accuracy obtained for CVD-3YFU is better than or equivalent to CVD-CR. (iii) SMOTE effect: Since AI requires a larger sample size for better training and accuracy prediction, we use SMOTE. Therefore, we hypothesize that the ML risk prediction using SMOTE $5 \mathrm{X}$ is superior to ML risk prediction without SMOTE. In continuation, we believe that the accuracy using SMOTE $5 \mathrm{X}$ for CVD-CR is superior to accuracy without SMOTE for CVD-CR. Further, the accuracy using SMOTE $5 \mathrm{X}$ for CVD-3YFU is superior to accuracy without SMOTE for CVD-3YFU. Finally, the accuracy using SMOTE $5 \mathrm{X}$ for ML-CVRC is superior to accuracy without SMOTE for ML-CCVRC. (iv) ML effect: lastly, we hypothesize that ML-based calculators for CVDCR and CVD-3YFU scenarios are better than CCVRC.

\section{Data preparation using SMOTE}

CVD risk detection is a two-class problem. The distribution of participants was imbalanced. Considering CVE as an endpoint, the numbers of patients at visit 1 and visit 2 (CVD-CR and CVD-3YFU) setups were only seven and eight, respectively. This imbalanced dataset in terms of endpoint would result in bias of predicting boundary separation towards the majority class and this affects the performance of ML classifiers. So, to overcome this imbalance class issue, we used a well-published and standardized technique called "synthetic minority oversampling technique (SMOTE)" [37]. SMOTE algorithm used for balancing the risk classes and for data augmentation followed a nearest-neighbour technique that generated non-overlapping synthetic samples for the minority class. After using five times SMOTE (SMOTE $5 \mathrm{X})(n=2710)$, the dataset was partitioned in two halves using a standardized two-fold cross-validation technique to train and evaluate the ML-based system.

\section{Overall machine learning architecture}

ML-based algorithms can learn and capture the non-linear, and more complex "information" which is available in the risk features and risk predictors (consisting of OBBM, LBBM, and CUSIP) when together taken as part of the input cohort features. ML uses the gold standards (CVE) along with the covariates (risk factors) to learn the behaviour and patterns in an offline mode to generate ML-based models. These are then used to transform the test patient covariates into a predicted risk. Such a framework is adopted for CVDCR setup and CVD-3YFU setu Since some of the covariates (features) are more powerful than other covariates, therefore, ML-based framework has the ability to orderly select features to increase the overall performance. Such ML-based calculators are benchmarked against CCVRC.

The ML architecture follows the standardized architecture where the ML offline training model is generated using the combination of training data and CVE as ground truth [38]. The CVD presence risk is predicted during the online process where the test patient data are transformed by the ML offline model. The accuracy of such a system is estimated using the $\mathrm{K} 2$ cross-validation $(\mathrm{CV})$ protocol, where the data sets are divided into two parts, $50 \%$ used for training and the remaining 50\% used for testing. This is implemented in the cyclic process where the test set is unique. Before $\mathrm{CV}$ execution and data partitioning, the system undergoes preprocessing of the data set using SMOTE algorithm. For optimization and preprocessing, we had used the Standard Scalar function for mapping the independent covariates in the range 0 to 1 . Principal component analysis [39] with pooling (PCA pooling) was used to select the most significant features. PCA pooling selects covariates according to the magnitude (from high to low in absolute values) of their coefficients. Followed by this, we used Label Encoder to encode class values as integers. The performance of the system is computed using the following parameters such as sensitivity, specificity, positive predictive value, negative predictive value, false-positive rate, false-negative rate, accuracy, and area under curve score. We benchmarked our ML-based calculator against CCVRC. The overall system architecture is shown in.

\section{Feature selection using two methods: PCA pooling and mutual information}

PCA pooling is a well-known feature selection technique $[39,40]$ used for minimizing the dimensionality of the data. On the other hand, mutual information [41] is a feature selection technique that shows which covariate is highly significant in predicting the target ground-truth label. The PCA pooling technique is used to increase the performance of the CVD-CR and CVD-3YFU by finding the best cluster order among the OBBM, LBBM, and CUSI The decreasing order of selecting features using PCA pooling is shown in Table A1 of Appendix A for both CVD-CR and CVD$3 Y F U$. The top covariate is the highest predictive power (LCB PLQ) and the bottom one is the least power (Total number of plaques). 
Table 1 Cluster Effect: without SMOTE for CVD-CR and CVD-3YFU

\begin{tabular}{|c|c|c|c|c|c|c|c|}
\hline \multirow[t]{2}{*}{$\mathrm{SN}$} & \multirow[t]{2}{*}{ Clusters } & \multicolumn{3}{|l|}{ CVD-CR } & \multicolumn{3}{|l|}{ CVD-3YFU } \\
\hline & & LDA & SVMrbf & $\mathrm{RF}$ & LDA & SVMrbf & $\mathrm{RF}$ \\
\hline 1 & Cluster 1 & $82.10 \pm 14.94 \%$ & $98.71 \pm 0.18 \%$ & $98.71 \pm 0.32 \%$ & $83.03 \pm 13.65 \%$ & $98.52 \pm 0.00 \%$ & $98.52 \pm 0.00 \%$ \\
\hline 2 & Cluster 2 & $84.69 \pm 14.21 \%$ & $98.71 \pm 0.18 \%$ & $98.71 \pm 0.32 \%$ & $95.02 \pm 3.51 \%$ & $98.52 \pm 0.00 \%$ & $98.52 \pm 0.00 \%$ \\
\hline 3 & Cluster 3 & $86.53 \pm 11.99 \%$ & $98.71 \pm 0.18 \%$ & $98.71 \pm 0.32 \%$ & $95.39 \pm 2.77 \%$ & $98.52 \pm 0.00 \%$ & $98.52 \pm 0.00 \%$ \\
\hline
\end{tabular}

$R F$ random forest; $S V M r b f$ support vector machine with radial basis function; $L D A$ linear discriminant analysis; $C V D$ - $C R$ cardiovascular diseasecurrent risk; $C V D-3 Y F U$ cardiovascular disease risk-three-year follow-up

\section{Training/Testing classifiers and performance evaluation metrics}

We used 3 types of classifiers such as random forest (RF) [42], support vector machine with the radial basis function (SVMrbf) [43, 44], and linear discriminant analysis (LDA) $[45,46]$ to perform CVD-CR and CVD-3YFU using the combination of 46 covariates and CVE (as ground truth) in the ML framework. The theory and the optimization parameters of these classifiers are shown in Appendix B. The performance evaluation metrics are shown in Appendix C. Further, we compare the ML calculators (RF, SVMrbf, and LDA) against the conventional calculators (FRS, SCORE, and ASCVD) $[23,47]$.

\section{Experimental protocol}

\section{Experiment 1: CVD risk prediction using cross-validation (PCA vs. MI)}

The objective of this experiment is to predict the CVD risk using the $\mathrm{K} 2$ type of $\mathrm{CV}$ protocol. In this protocol, the patient data are equally divided into $50 \%$ training and $50 \%$ testing; keep the ratio of CVD to non-CVD patients. The training model is generated (as per Fig. 2) using PCA training-based features, and then the model is applied to the test features to predict the CVD risk. A confusion matrix is determined along with the AUC as part of the performance. The objective is to compare and contrast the feature effect of the PCA vs. MI feature selection methods using the $\mathrm{K} 2$ $\mathrm{CV}$ protocol.

\section{Experiment 2: Study the effect of features on CVD risk prediction (Cluster Effect)}

The objective of this experiment is to study the effect of the features on CVD risk. The accuracy, performance evaluation matrices, and ROC are performed for all three clusters using PCA pooling, K2 CV protocol, with and without SMOTE $5 \mathrm{X}$ for both CVD-CR and CVD-3YFU. The accuracy of all three clusters was compared to study the effect of the feature.

\section{Results}

This section presents the accuracy results of the three classifiers for the CVD-CR and CVD-3YFU experiment while using PCA and MI-based feature extraction techniques. In the second part of the results, we present the cluster effect in SMOTE framework and compare it with without SMOTE. The second part of Sect. 5 presents the PE for (a) generation of performance matrix parameters and (b) ROC analysis. Finally, part three of the section demonstrate the statistical tests such as Tukey [48] and Shapiro-Wilks [49] for validating the significant covariates computed from Chi square $\left(\chi^{2}\right)$ and ANOVA test) [50].

\section{Baseline characteristics}

In each participant, 46 variable or CVD risk factors were assessed and were partitioned into 3 clusters. (i) cluster 1 included OBBM (see Table A2 of Appendix A) consisting of baseline characteristics from row number R1 to row number R13. This consisted of risk factors such as sex, age, weight, height, body mass index (BMI), average systolic blood pressure (AvSBP), average diastolic blood pressure (AvDBP), heart rate (HR), family history of coronary artery disease (Family His CAD), current smoker, hypertension, diabetes, and hyperlipidemia. (ii) cluster 2 included both OBBM and LBBM (see Table A2 baseline characteristics from row number R1 to row number R34), and (iii) cluster 3 included OBBM, LBBM, and CUSIP (Table A2 baseline characteristics from row number R1 to row number R46). There are two columns in the participant's characteristics: Part A consists of data recorded at visit 1 and Part B consist of the same variable recorded at visit 2 (after 3-year followup). Statistical tests were used to compute the $p$ value. Note that in our cohort, we observed that only one more patient was added to the CVD pool from visit 1 to visit 2 .

A. Significant CVD risk factors and established CVD as ground truth at visit 1

We observed five significant covariates which had $p$ value $<0.05$ in visit 1 with the presence of CVD events as ground truth (CVD-CR): (R10) current smoker with 


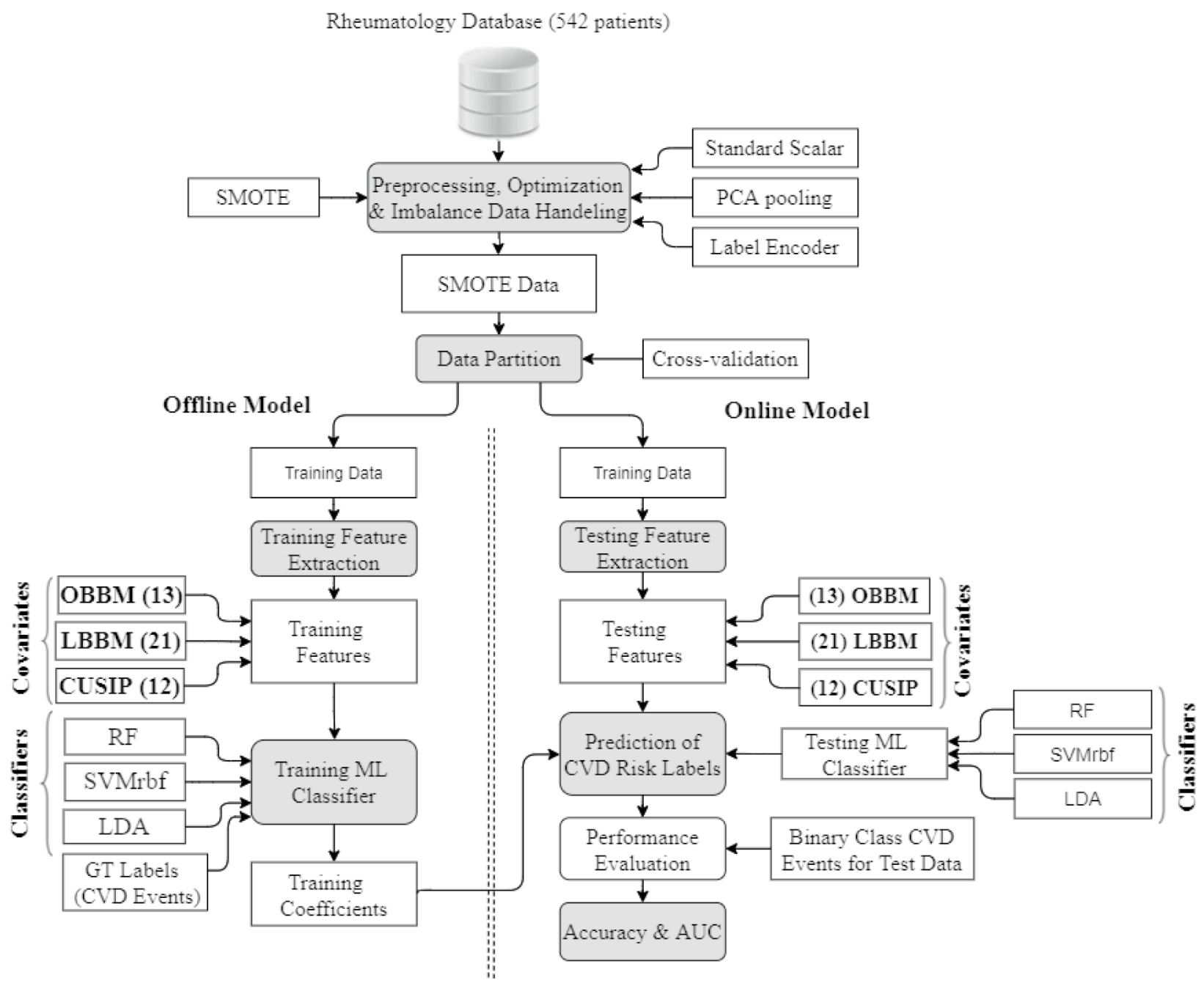

Fig. 2 Overall system architecture for ML-based CVD risk stratification system. $M L$ machine learning; $A U C$ area-under-the-curve; $O B B M$ office-based biomarkers; $L B B M$ laboratory-based biomarker; $C U S I P$ carotid ultrasound image phenotype; $R F$ random forest;

$p$ value 0.006 , (R16) c-reactive protein (CRP) with $\mathrm{p}$ value 0.018 , (R17) cholesterol (Chol) with $p$ value 0.023 , (R32) low-density lipoprotein(LDL) with $p$ value 0.026 and (R33) urea (UA) with p value 0.033 .

B. Significant CVD risk factors and established CVD as ground truth at visit 2

There were six significant covariates which had $p$ value $<0.05$ in visit 2 with the presence of CVD as ground truth (CVD-CR): (R10) current smoker with $p$ value 0.007, (R16) CRP with $p$ value 0.008 , (R17) Chol with $p$ value 0.010 , (R32) LDL with $\mathrm{p}$ value 0.017 , (R33) UA with $p$ value 0.040 , and (R34) thyroid-stimulating hormone (TSH) with $\mathrm{p}$ value 0.013 .
SVMrbf support vector machine with radial basis function; $L D A$ linear discriminant analysis; $G T$ ground truth; $C V D$ cardiovascular disease

\section{Accuracy for CVD-CR and CVD-3YFU (MI vs. PCA pooling)}

The accuracy comparison of Mutual Information vs. PCA pooling is shown in Table A3 of Appendix A. The mean accuracy for PCA pooling (97.98\%) is superior to the mean accuracy of MI (97.06\%) for CVD-CR and the mean accuracy of PCA pooling (97.48\%) is also superior to the mean accuracy of MI (97.34\%) for CVD-3YFU. All the three classifiers had greater accuracy for PCA pooling than MI for both the experiments (CVD-CR and CVD-3YFU). Therefore, we had proved that feature selection using PCA pooling is better than MI. 
Fig. 3 Mean Accuracy (\%) using SMOTE $5 \mathrm{X}$ for CVD-CR. $R F$ : random forest, $S V M r b f$ : support vector machine with radial basis function, $L D A$ : linear discriminant analysis, Cluster 1: OBBM, Cluster 2: fusion of OBBM, and LBBM, and Cluster 3: fusion of OBBM, LBBM, and CUSIP

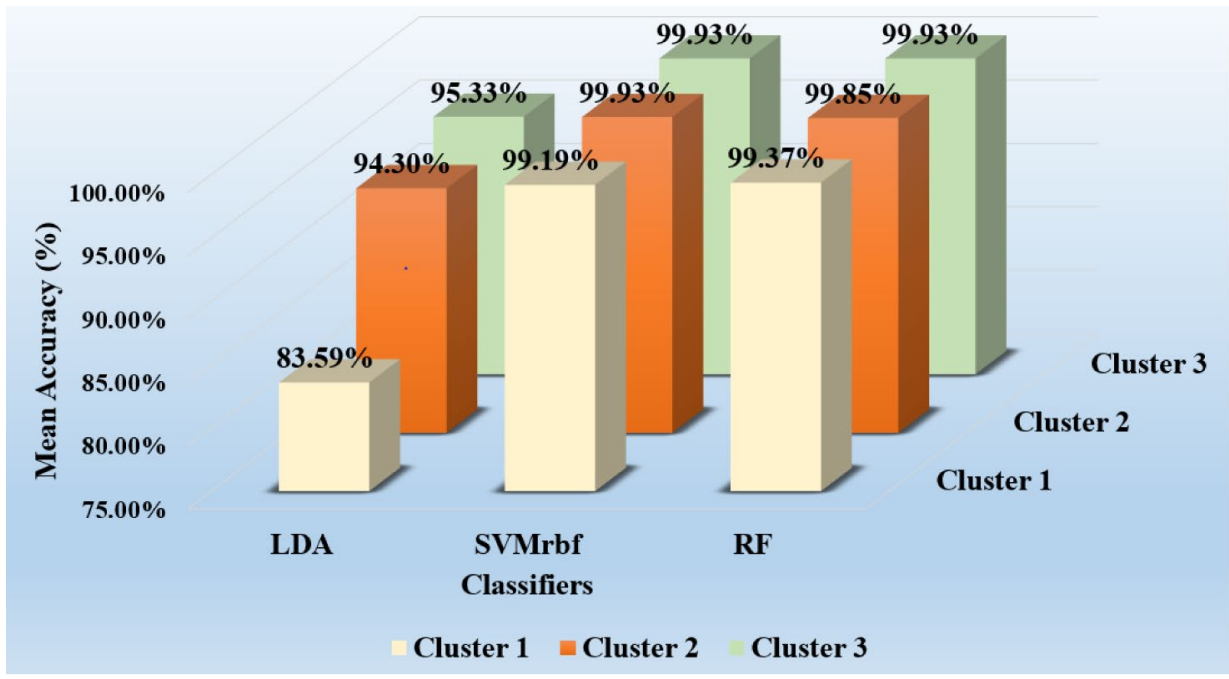

Fig. 4 Mean Accuracy (\%) using SMOTE $5 \mathrm{X}$ for CVD3 YFU. $R F$ random forest, SVMrbf support vector machine with radial basis function, $L D A$ linear discriminant analysis, Cluster 1 OBBM, Cluster 2 fusion of OBBM, and LBBM, and Cluster 3 fusion of OBBM, LBBM, and CUSIP

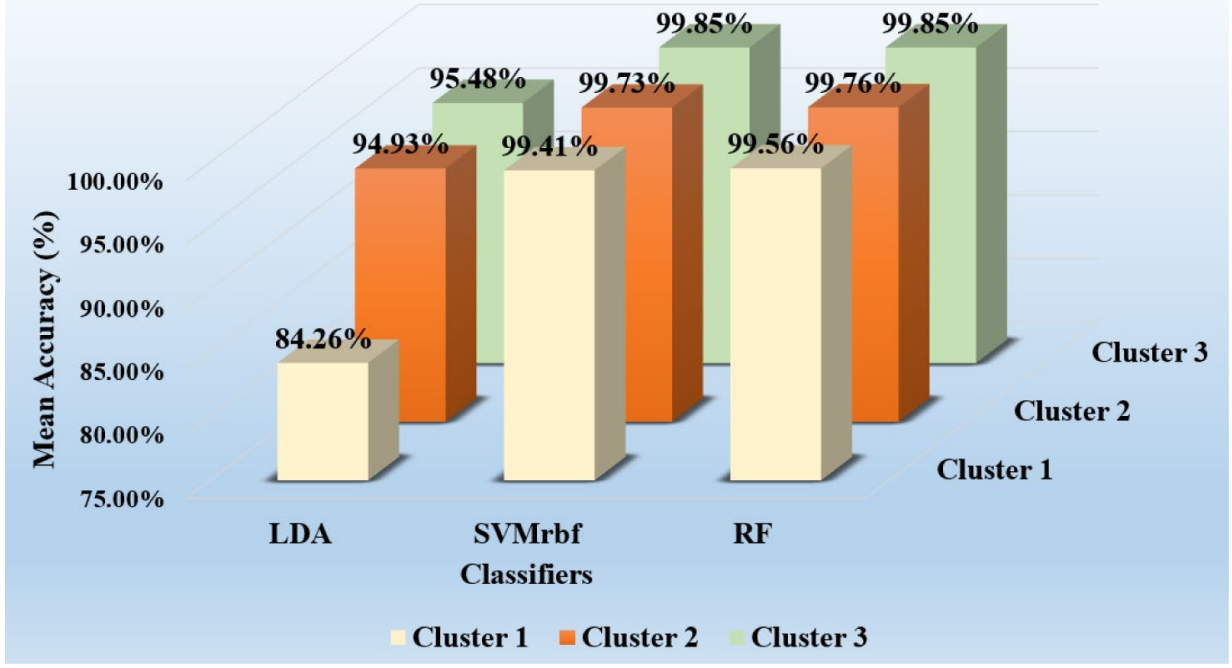

\section{Effect of clusters on CVD-CR and CVD-3YFU}

This section presents the effect of the features when taken in the form of clusters. In Fig. 3, the bar chart shows the mean accuracy (in \%) using SMOTE 5X for CVD-CR setup, while Fig. 4 shows the mean accuracy (in \%) using SMOTE 5X for CVD-3YFU setu Both bar charts show the distribution of accuracy (z-axis) for the three classifiers (X-axis), for the corresponding clusters (Y-axis). Note that cluster 1 is OBBM, cluster 2 is OBBM combined with LBBM, while cluster 3 is OBBM, LBBM, and CUSI The bar chart for CVD-CR in Fig. 3 clearly shows the increase in the accuracy due to an increase in covariate strengths (cluster $3>$ cluster $2>$ cluster 1). This has the same behaviour in Fig. 4 for CVD-3YFU. This validates our hypothesis that with an increase in information due to covariate, all classifiers show better performance. Table 1 shows the cluster effect under non-SMOTE conditions, while Table 2 shows the cluster effect under SMOTE conditions.

\section{Performance evaluation}

This section has two parts: Part A presents the performance metrics of three classifiers corresponding to the three clusters in SMOTE 5X framework for CVD-CR and CVD3 YFU, respectively. Part B shows the ROC curves for six classifiers (three ML and three CCVRC) in SMOTE $5 \mathrm{X}$ framework for cluster 1, cluster 2, and cluster 3 under CVDCR condition and CVD-3YFU.

Table 3 shows the performance matrices (PE) for ML vs. CCVRC calculators corresponding to three clusters. The PE metrics consists of the following attributes: sensitivity, specificity, false-positive rate (FPR), false-negative rate (FNR), positive predictive value (PPV), negative predictive value (NPV), accuracy, and area-under-curve (AUC) along with their $\mathrm{p}$ value and its ranges. This is represented by column $\mathrm{C} 1$ to column $\mathrm{C} 11$ in Table 3 . Note that these metrics are computed in SMOTE $5 \mathrm{X}$ framework for the CVD-CR 
condition, while Table 4 shows the computation in SMOTE $5 \mathrm{X}$ for CVD-3YFU.

\section{ROC comparison between three types of ML vs. three types of CCVRC for all three clusters}

A comparison between all three ML classifiers (LDA, SVMrbf, and RF) against three CCVRC (FRS, SCORE, and ASCVD) for cluster $1(\mathrm{OBBM})$, cluster $2(\mathrm{OBBM}+\mathrm{LBBM})$, and cluster $3(\mathrm{OBBM}+\mathrm{LBBM}+\mathrm{CUSIP})$ using SMOTE $5 \mathrm{X}$ framework are shown in Fig. 5(a, b, c) corresponding to CVD-CR). Similar ROC curves can be seen in Fig. 6(a, b, c) for CVD-3YFU. The ML-based classifiers performed better than the CCVRC and the AUC score of all three ML-based classifiers $(0.98, p<0.001)$ were greater than the AUC score $(0.76, p<0.001)$ of all three CCVRCs for all clusters.

\section{Validation of significant covariates using Tukey/ Shapiro-Wilk Tests for CVD risks}

In baseline characteristics, Chi square $\left(\chi^{2}\right)$ test [50] was used to compare categorical covariates and the ANOVA test [51] was used for continuous covariates. Tukey's range test, also known as "Tukey's honestly significant difference (HSD) posthoc test" [48] was used to study the multiple comparisons of CVD risk for both CVD-CR and CVD-3YFU (see section S1 of the Supplementary material). Tukey's test always used only those covariates that showed a highly significant association with CVD-CR and CVD-3YFU. Shapiro-Wilk test [49] (Table A4), a nonparametric test was used to study the normality of the continuous risk covariates. All the continuous covariates were manifest as mean \pm SD and categorical covariates were expressed as percentages. All the statistical tests were two-tailed as the significance cut-off is $<0.05$. There were five significant covariates for the baseline characteristics in CVD-CR and six significant covariates in CVD-3YFU. Shapiro-Wilk tests were needed to assure that all the significant covariates from baseline characteristics should also be in the Shapiro-Wilk test. Tukey HSD posthoc is needed to show that the $p$ value from baseline should be the same as $p$ values from Tukey's test for the binary-class CVD risk. The validation of significant covariates using Tukey HSD post hoc and Shapiro-Wilk test is shown in Table 5 (for CVD-CR) and Table 6 (for CVD-3YFU), which signifies that the covariates which had significant cut-off $p<0.05$ from baseline characteristics are highly significant and validated.

\section{Scientific validation}

The validation of the CVD risk prediction system can be done by considering a validation database whose results are known prior. For our system, we used a coronary artery database consisting of 500 subjects having 39 covariates. The test participants had a carotid ultrasound which was conducted at the time of the angiogram [52]. There were 298 participants who had an angiographic score of high-risk and 202 participants had no-risk. The validation dataset for all participants had a mean age of $64.49 \pm 10.6$ years (ranging from 29 to 95 years), $69.8 \%$ were males, $67.59 \%$ hypertension, and $57.59 \%$ hyperlipidemia. The baseline characteristic of the validation coronary dataset for 500 test participants is shown in Table A5. There were 12 significant covariates that had a $p$ value $<0.05$. We had used a two-fold crossvalidation protocol (K2) along with PCA with pooling for best feature selection.

The performance of the cross-validation accuracy for all three classifiers (LDA, SVMrbf, and RF), for two-fold cross-validation (K2) on the validation coronary dataset, is shown in Table 7. In the validation dataset, the ML-based classifiers are also superior to the CCVRC calculators. The brief accuracy comparison between without SMOTE and with SMOTE 5X is shown in Table 7. In the validation coronary dataset, SVMrbf is shown the highest performance of $84.76 \%$ with SMOTE 5X. A brief comparison of the AUC score is shown in Table 8. Figure 7 shows the ROC curve for SVMrbf and RF in SMOTE 5X framework, both having the same value of 0.92 .

Our validation study had similar behaviour as the experimental database showing: (i) superior performance of the ML-based calculators against CCVRC calculators (Fig. 7), (ii) the performance of ML-based calculators with SMOTE $5 \mathrm{X}$ is superior to the performance of the ML-based calculators without SMOTE (Table 7), (iii) AUC score of ML-based

Table 2 Cluster Effect: with SMOTE 5X for CVD-CR and CVD-3YFU

\begin{tabular}{|c|c|c|c|c|c|c|c|}
\hline \multirow[t]{2}{*}{$\mathrm{SN}$} & \multirow[t]{2}{*}{ Clusters } & \multicolumn{3}{|l|}{ CVD-CR } & \multicolumn{3}{|l|}{ CVD-3YFU } \\
\hline & & LDA & SVMrbf & $\mathrm{RF}$ & LDA & SVMrbf & $\mathrm{RF}$ \\
\hline 1 & Cluster 1 & $83.59 \pm 0.19 \%$ & $99.19 \pm 0.22 \%$ & $99.37 \pm 0.56 \%$ & $84.26 \pm 0.26 \%$ & $99.41 \pm 0.07 \%$ & $99.56 \pm 0.00 \%$ \\
\hline 2 & Cluster 2 & $94.30 \pm 1.48 \%$ & $99.93 \pm 0.00 \%$ & $99.85 \pm 0.00 \%$ & $94.93 \pm 2.19 \%$ & $99.73 \pm 0.07 \%$ & $99.76 \pm 0.04 \%$ \\
\hline 3 & Cluster 3 & $95.33 \pm 1.48 \%$ & $99.93 \pm 0.07 \%$ & $99.93 \pm 0.07 \%$ & $95.48 \pm 1.78 \%$ & $99.85 \pm 0.07 \%$ & $99.85 \pm 0.07 \%$ \\
\hline
\end{tabular}

$R F$ random forest; $S V M r b f$ support vector machine with radial basis function; $L D A$ linear discriminant analysis; $C V D$ - $C R$ cardiovascular diseasecurrent risk; $C V D$-3YFU cardiovascular disease risk-three-year follow-up 


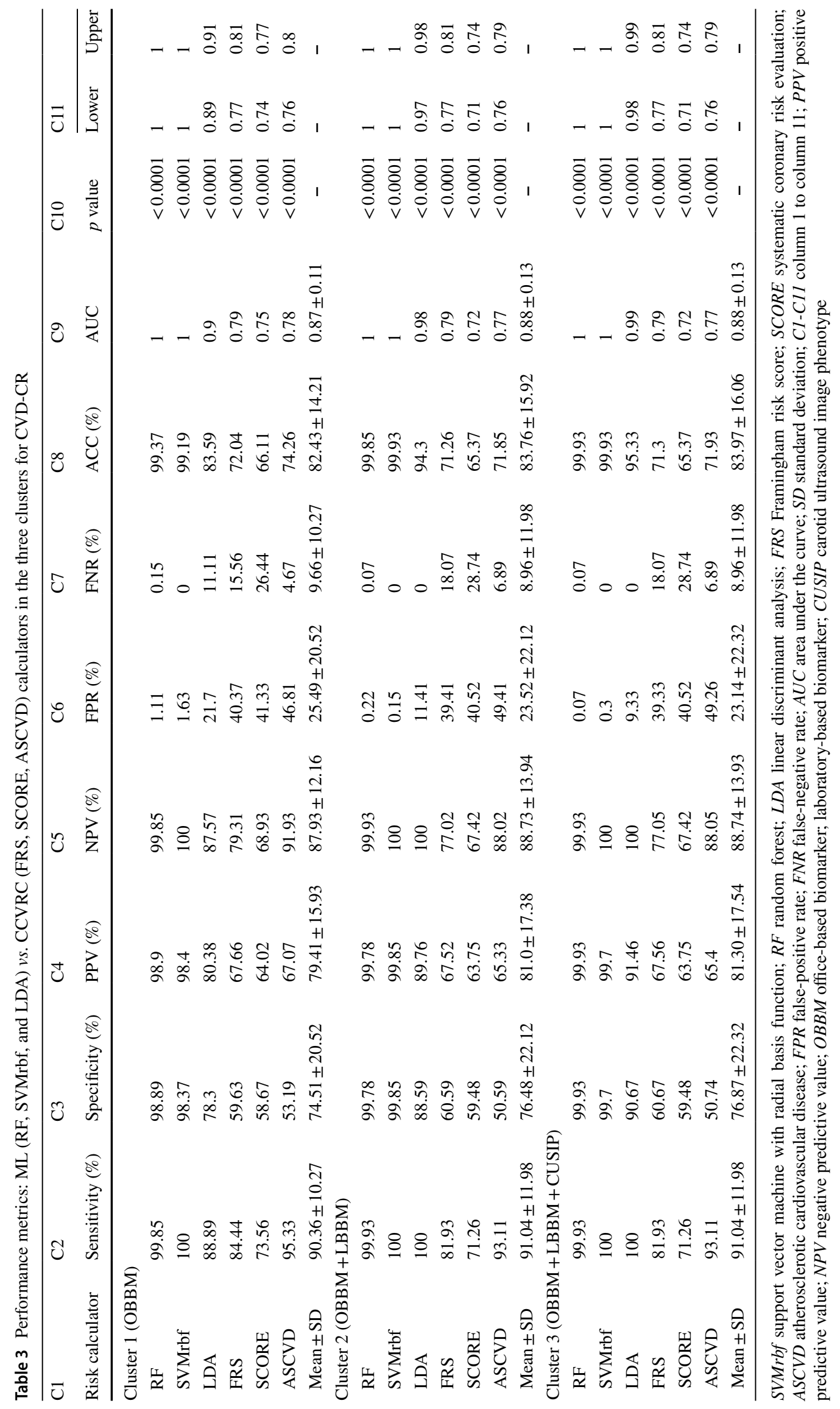




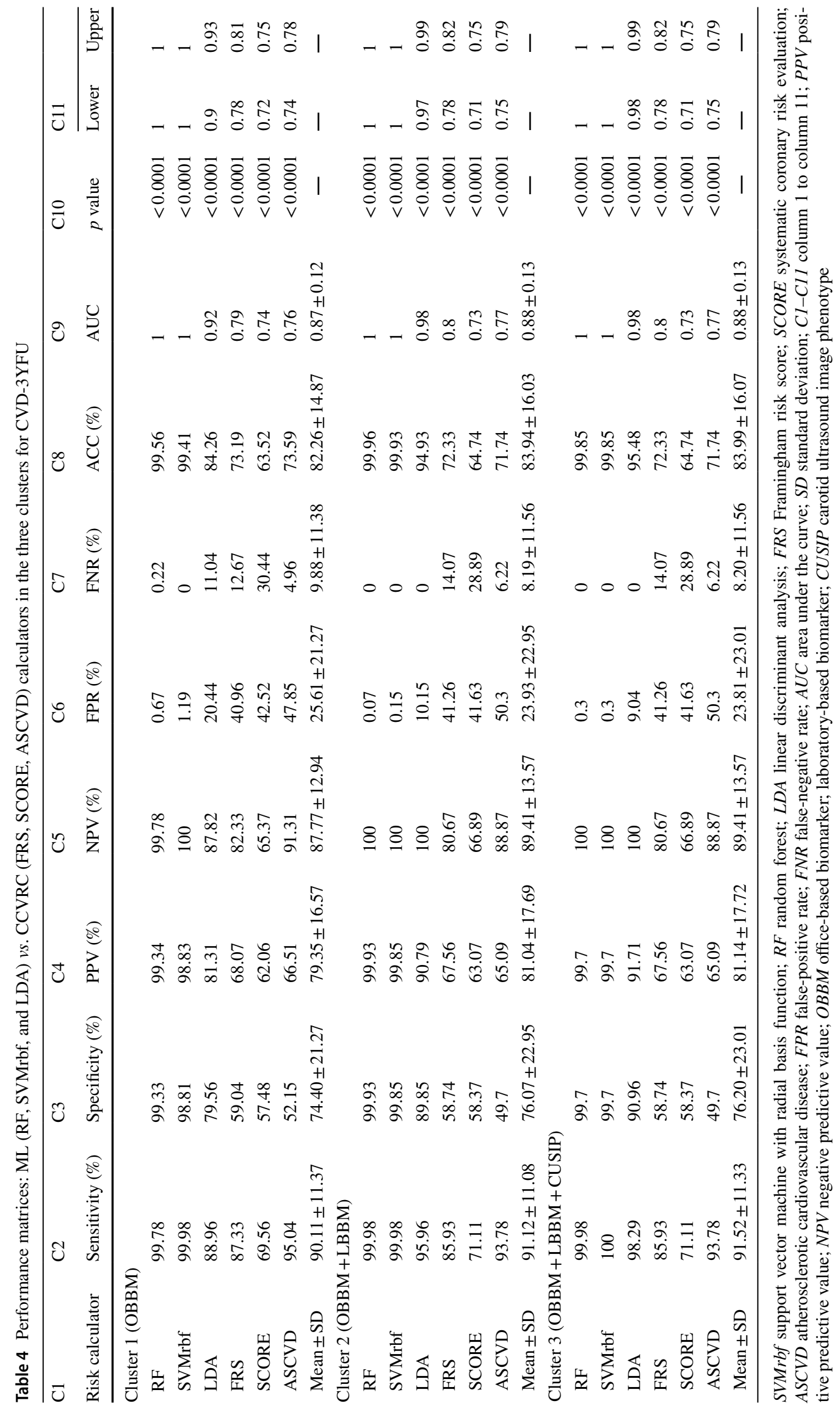


(a)

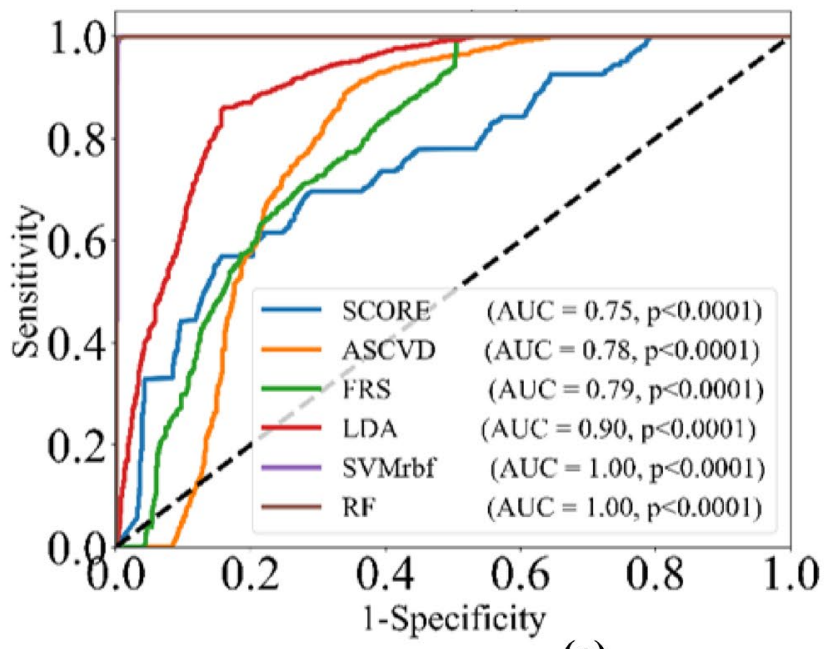

(b)

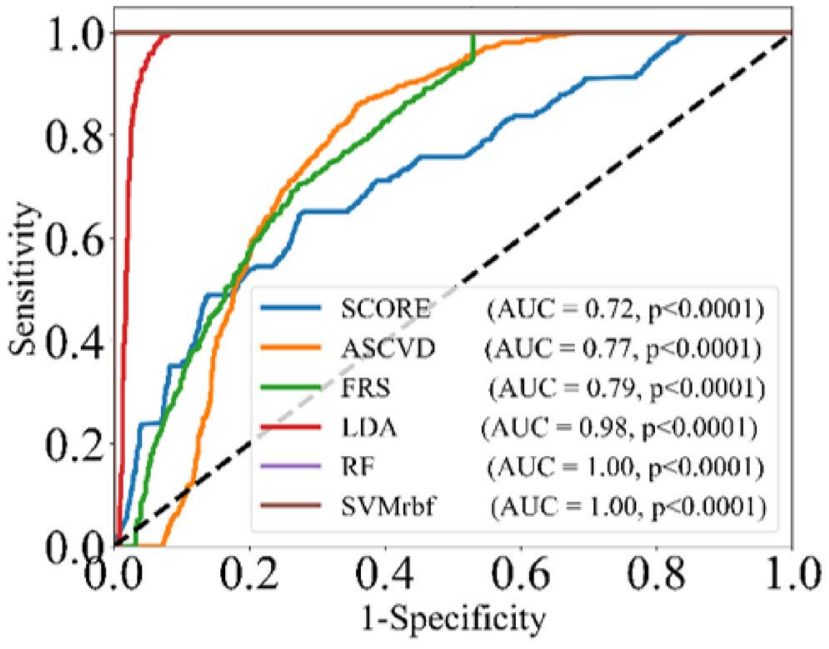

(c)

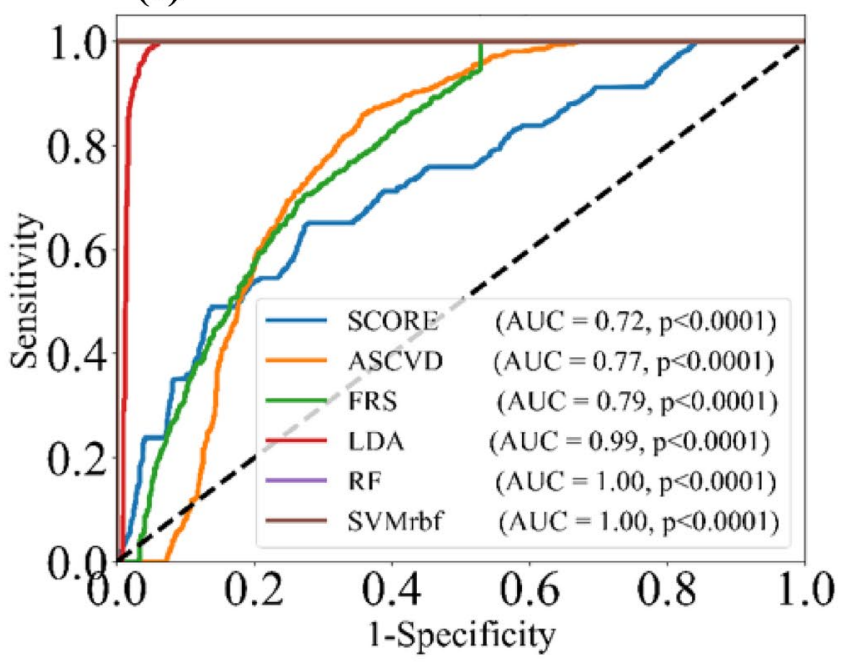

Fig. 5 AUC curve for (a) Cluster 1, (b) Cluster 2, and (c) Cluster 3, respectively for CVD-CR; $R F$ random forest; $S V M r b f$ support vector machine with radial basis function; $L D A$ linear discriminant analysis;

calculators with SMOTE $5 \mathrm{X}$ is superior to ML-based calculators without SMOTE (Table 8). Thus, the validation proved our hypothesis to be true.

\section{Discussions}

This is the first pilot study of its kind where the CVD presence can be detected using current covariates using twotime points (i) visit 1 current CVE as ground truth, and (ii) visit 2 (3-year follow-up) CVE as ground truth. The AtheroEdge ${ }^{\mathrm{TM}} 3.0$ (ML) used 46 covariates having both nonRA and RA patients. Using SMOTE framework and feature extraction paradigms such as PCA pooling or MI, our system
$A U C$ area-under-curve; FRS Framingham Risk Score; SCORE systematic coronary risk evaluation, $A S C V D$ atherosclerotic cardiovascular disease

was able to generate superior performance with cross-validation $\mathrm{K} 2$ protocol while validating the following hypothesis: (i) Cluster Effect: The ML classifiers showed superior performance when carotid image phenotypes were fused with conventional and laboratory-based risk factors, unlike without carotid image phenotypes(Table 9); (ii) SMOTE Effect: CVD detection was superior in SMOTE framework, unlike in non-SMOTE framework, both when using current CVE (Table 10) or 3 YFU CVE (Table 11) (iii) Followup Effect: CVD risk prediction using 3-year CVE showed equal or superior to CVD risk prediction with current CVE (Table 12); (iv) ML Effect: ML-based calculators showed superior performance compared to CCVRC (Table 13). The overall ML-based framework is automated and uses the 
(a)

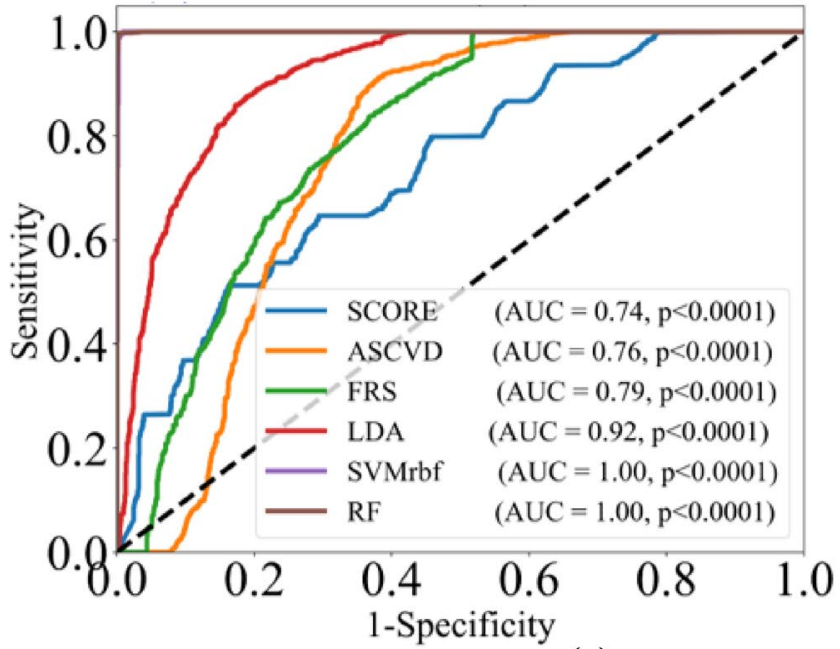

(b)

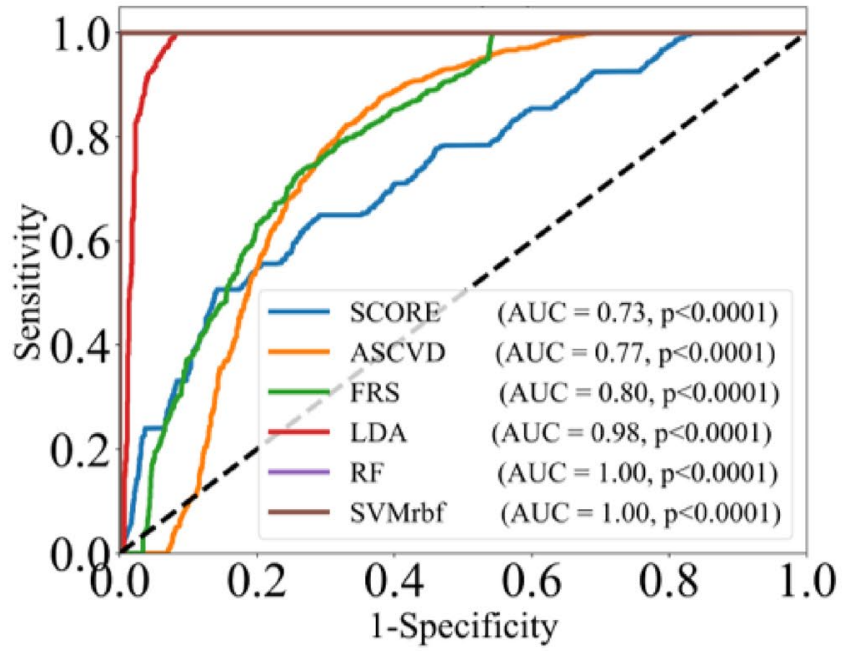

(c)

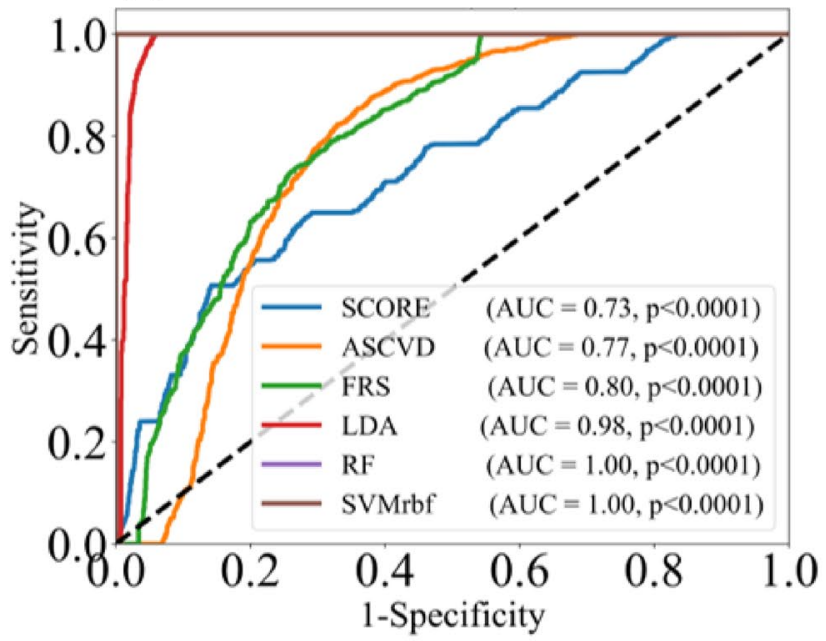

Fig. 6 AUC curve for (a) Cluster 1, (b) Cluster 2, and (c) Cluster 3, respectively for CVD-3YFU. RF: random forest; SVMrbf: support vector machine with radial basis function; LDA: linear discriminant analysis; AUC: area-under-curve; FRS Framingham Risk Score; $S C O R E$ systematic coronary risk evaluation, ASCVD atherosclerotic cardiovascular disease
Table 5 Validating significant covariates using Tukey's HSD \& Shapiro-Wilk test for CVD-CR

\begin{tabular}{llllll}
\hline SN & Covariate Type & Tukey's Test & Shapiro-Wilk Test & $\begin{array}{l}\text { Baseline (Chi-square } \\
\text { and ANOVA) }\end{array}$ & Validated \\
\hline 1 & Curr-Smoker & 0.006 & $<0.0001$ & 0.006 & $\checkmark$ \\
2 & CRP & 0.018 & $<0.0001$ & 0.018 & $\checkmark$ \\
3 & Chol & 0.023 & $<0.0001$ & 0.023 & $\checkmark$ \\
4 & LDL & 0.026 & $<0.0001$ & 0.026 & $\checkmark$ \\
5 & UA & 0.033 & $<0.0001$ & 0.033 & $\checkmark$ \\
\hline
\end{tabular}

Curr-Smoker Current Smoker; CRP C-reactive protein; Chol cholesterol; LDL low-density lipoprotein; UA uric acid
Scikit-learn technique [53], which is an open-source library using Python. Our ML-based framework is user-friendly, feasible and very flexible to run.

\section{Benchmarking}

There are not many AI techniques for the CVD risk assessment in cohorts that include rheumatology patients. 
Table 6 Validating significant covariates using Tukey's HSD \& Shapiro-Wilk test for CVD$3 \mathrm{YFU}$

\begin{tabular}{llllll}
\hline SN & Covariate type & Tukey's test & Shapiro-Wilk test & $\begin{array}{l}\text { Baseline (Chi square } \\
\text { and ANOVA) }\end{array}$ & Validated \\
\hline 1 & Curr-Smoker & 0.007 & $<0.0001$ & 0.007 & $\checkmark$ \\
2 & CRP & 0.008 & $<0.0001$ & 0.008 & $\checkmark$ \\
3 & Chol & 0.010 & $<0.0001$ & 0.010 & $\checkmark$ \\
4 & LDL & 0.017 & $<0.0001$ & 0.017 & $\checkmark$ \\
5 & UA & 0.040 & $<0.0001$ & 0.040 & $\checkmark$ \\
6 & TSH & 0.013 & $<0.0001$ & 0.013 & $\checkmark$ \\
\hline
\end{tabular}

Curr-Smoker Current Smoker; CRP C-reactive protein; Chol cholesterol; LDL low-density lipoprotein; UA uric acid
Table $7 \mathrm{CV}$ accuracy (\%) for coronary data without and with SMOTE $5 \mathrm{X}$ for $\mathrm{K} 2$

\begin{tabular}{lllll}
\hline SN & Classifiers & Without & With SMOTE & \% Improvement \\
& & SMOTE & $5 \mathrm{X}$ & \\
\hline 1 & LDA & $64.80 \pm 0.00 \%$ & $70.48 \pm 0.95 \%$ & 0.08 \\
2 & SVMrbf & $65.20 \pm 3.20 \%$ & $84.76 \pm 1.59 \%$ & 0.23 \\
3 & RF & $65.20 \pm 3.20 \%$ & $81.75 \pm 1.27 \%$ & 0.20 \\
\hline
\end{tabular}

$R F$ random forest; $S V M r b f$ support vector machine with radial basis function; $L D A$ linear discriminant analysis; $5 X$ five times

Table 8 AUC score for coronary data without and with SMOTE 5X for $\mathrm{K} 2$

\begin{tabular}{lllll}
\hline SN & Classifiers & $\begin{array}{l}\text { Without } \\
\text { SMOTE }\end{array}$ & $\begin{array}{l}\text { With } \\
\text { SMOTE 5X }\end{array}$ & \% Improvement \\
\hline 1 & LDA & 0.67 & 0.76 & 0.11 \\
A2 & SVMrbf & 0.53 & 0.92 & 0.42 \\
3 & RF & 0.64 & 0.92 & 0.30 \\
\hline
\end{tabular}

$R F$ random forest; $S V M r b f$ support vector machine with radial basis function; $L D A$ linear discriminant analysis; $5 X$ five times

However, AI techniques have been used to assess the severity of RA without considering the CVD risk [54-56]. Some of the reasons were that there is not much trend between people to estimate rheumatoid arthritis in patients due to bone join, and there is not much direct link between heart diseases on bone problems. This kind of research needs very dedicated follow-up and more money is needed for bone pain than for heart diseases. Because of that weak link, there are very few papers on rheumatology patients, that is why there are only seven patients in our rheumatology database who had CVD risk assessment $[17,57]$.

Six out of ten authors have used SVM classifiers for the prediction of CVD/stroke risk assessment. Further, five out of ten authors used follow-up CVE. Seven out of ten had used conventional cardiovascular risk factors as feature types, and three out of ten had used PCA-based technique for the selection of features. The similarity between other authors' studies and our study is that we had used follow-up

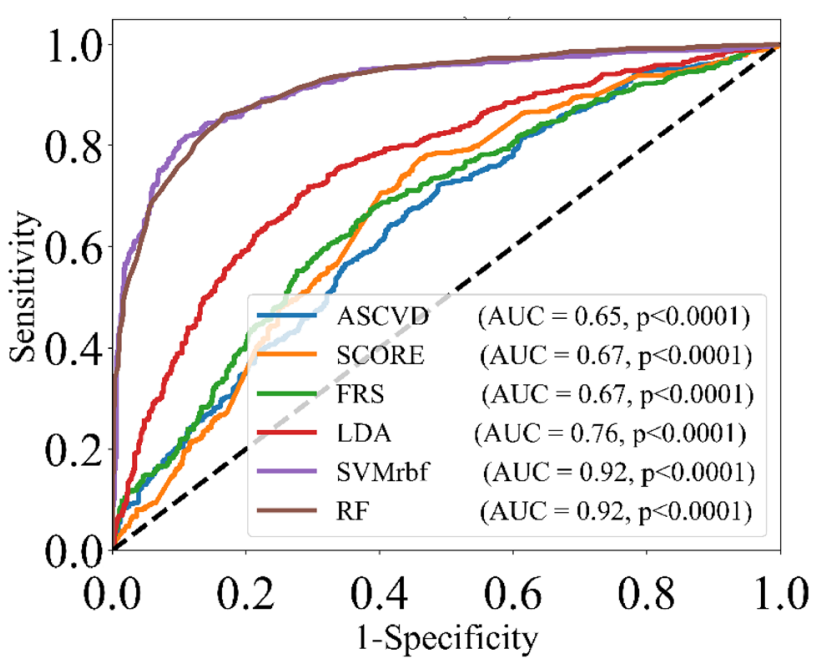

Fig. 7 AUC curve for K2 and SMOTE 5X for the validation coronary dataset. RF: random forest; SVMrbf: support vector machine with radial basis function; $L D A$ linear discriminant analysis; $A U C$ areaunder-curve; FRS Framingham Risk Score; SCORE systematic coronary risk evaluation; $A S C V D$ atherosclerotic cardiovascular disease

CVE, PCA-based technique for features selection, and SVM classifier for the prediction of CVD risk assessment (see Table 14, benchmarking).

A series of ML-based publications were proposed by Suri and Jamthikar, which are being summarized as follows: Jamthikar et al. [58] showed the usage of ML-based system (called AtheroRisk-integrated from AtheroPoint, Roseville, CA) performs better than the conventional calculator using PCA-based feature extraction system in K10 paradigm. The database used 13 current risk factors and 34 carotid image-based phenotypes, totalling 47 covariates. The authors used event-equivalent gold standard (EEGS) as percentage stenosis in the carotid artery. The ML-framework used RF classifier leading an AUC of ML system: 0.80 (95\% CI: 0.77-0.84) against AUC for CCVRC: 0.68 (95\% CI: $0.64-0.72$ ), showing an improvement of $18 \%$. The methodology neither used SMOTE, nor the ground truth had CVE. Further, the system did not consist of RA patients. In another 
Table 9 Hypothesis: Cluster effect (Cluster3 > Cluster2 > Cluster1) using accuracy with SMOTE 5X

\begin{tabular}{|c|c|c|c|c|c|c|}
\hline \multirow[t]{2}{*}{ Clusters } & \multicolumn{3}{|l|}{ CVD-CR } & \multicolumn{3}{|l|}{ CVD-3YFU } \\
\hline & LDA & SVMrbf & $\mathrm{RF}$ & LDA & SVMrbf & $\mathrm{RF}$ \\
\hline Cluster $1(\mathrm{C} 1)$ & $83.59 \pm 0.19 \%$ & $99.19 \pm 0.22 \%$ & $99.37 \pm 0.56 \%$ & $84.26 \pm 0.26 \%$ & $99.41 \pm 0.07 \%$ & $99.56 \pm 0.00 \%$ \\
\hline Cluster 2 (C2) & $94.30 \pm 1.48 \%$ & $99.93 \pm 0.00 \%$ & $99.85 \pm 0.00 \%$ & $94.93 \pm 2.19 \%$ & $99.73 \pm 0.07 \%$ & $99.76 \pm 0.04 \%$ \\
\hline Cluster 3 (C3) & $95.33 \pm 1.48 \%$ & $99.93 \pm 0.07 \%$ & $99.93 \pm 0.07 \%$ & $95.48 \pm 1.78 \%$ & $99.85 \pm 0.07 \%$ & $99.85 \pm 0.07 \%$ \\
\hline $\begin{array}{l}\text { Hypothesis } \\
\qquad(\mathrm{C} 3>\mathrm{C} 2>\mathrm{C} 1)\end{array}$ & $\checkmark$ & $\checkmark$ & $\checkmark$ & $\checkmark$ & $\checkmark$ & $\checkmark$ \\
\hline
\end{tabular}

$R F$ random forest; SVMrbf: support vector machine with radial basis function; $L D A$ linear discriminant analysis; $C V D$ - $C R$ cardiovascular disease-current risk; $C V D$-3YFU cardiovascular disease risk-three-year follow-up

Table 10 Hypothesis: SMOTE 5X performance is superior tow/o SMOTE for CVD-CR

\begin{tabular}{|c|c|c|c|c|c|c|c|}
\hline \multirow[t]{2}{*}{ Clusters } & \multicolumn{3}{|l|}{ W/o SMOTE } & \multicolumn{3}{|c|}{ With SMOTE $5 X$} & \multirow[t]{2}{*}{ Hypothesi } \\
\hline & LDA & SVMrbf & $\mathrm{RF}$ & LDA & SVMrbf & $\mathrm{RF}$ & \\
\hline Cluster 1 & $82.10 \pm 14.94 \%$ & $98.71 \pm 0.18 \%$ & $98.71 \pm 0.32 \%$ & $83.59 \pm 0.19 \%$ & $99.19 \pm 0.22 \%$ & $99.37 \pm 0.56 \%$ & $\checkmark$ \\
\hline Cluster 2 & $84.69 \pm 14.21 \%$ & $98.71 \pm 0.18 \%$ & $98.71 \pm 0.32 \%$ & $94.30 \pm 1.48 \%$ & $99.93 \pm 0.00 \%$ & $99.85 \pm 0.00 \%$ & $\checkmark$ \\
\hline Cluster 3 & $86.53 \pm 11.99 \%$ & $98.71 \pm 0.18 \%$ & $98.71 \pm 0.32 \%$ & $95.33 \pm 1.48 \%$ & $99.93 \pm 0.07 \%$ & $99.93 \pm 0.07 \%$ & $\checkmark$ \\
\hline
\end{tabular}

$R F$ random forest; $S V M r b f$ support vector machine with radial basis function; $L D A$ linear discriminant analysis; $C V D$ - $C R$ cardiovascular diseasecurrent risk; $C V D$-3YFU cardiovascular disease risk-three-year follow-up

Table 11 Hypothesis: SMOTE 5X performance are superior to w/o SMOTE for CVD-3YFU

\begin{tabular}{|c|c|c|c|c|c|c|c|}
\hline \multirow[t]{2}{*}{ Clusters } & \multicolumn{3}{|l|}{ W/o SMOTE } & \multicolumn{3}{|c|}{ With SMOTE 5X } & \multirow[t]{2}{*}{ Hypothesis } \\
\hline & LDA & SVMrbf & $\mathrm{RF}$ & LDA & SVMrbf & $\mathrm{RF}$ & \\
\hline Cluster 1 & $83.03 \pm 13.65 \%$ & $98.52 \pm 0.00 \%$ & $98.52 \pm 0.00 \%$ & $84.26 \pm 0.26 \%$ & $99.41 \pm 0.07 \%$ & $99.56 \pm 0.00 \%$ & $\checkmark$ \\
\hline Cluster 2 & $95.02 \pm 3.51 \%$ & $98.52 \pm 0.00 \%$ & $98.52 \pm 0.00 \%$ & $94.93 \pm 2.19 \%$ & $99.73 \pm 0.07 \%$ & $99.76 \pm 0.04 \%$ & $\checkmark$ \\
\hline Cluster 3 & $95.39 \pm 2.77 \%$ & $98.52 \pm 0.00 \%$ & $98.52 \pm 0.00 \%$ & $95.48 \pm 1.78 \%$ & $99.85 \pm 0.07 \%$ & $99.85 \pm 0.07 \%$ & $\checkmark$ \\
\hline
\end{tabular}

$R F$ random forest; $S V M r b f$ support vector machine with radial basis function; $L D A$ linear discriminant analysis; $C V D$ - $C R$ cardiovascular diseasecurrent risk; $C V D$-3YFU cardiovascular disease risk-three-year follow-up

Table 12 Hypothesis: performance of CVD-3YFU is better or equivalent to CVD-CR

\begin{tabular}{|c|c|c|c|c|c|c|c|}
\hline \multirow[t]{2}{*}{ Clusters } & \multicolumn{3}{|l|}{ CVD-CR } & \multicolumn{3}{|l|}{ CVD-3YFU } & \multirow[t]{2}{*}{ Hypothesis } \\
\hline & LDA & SVMrbf & $\mathrm{RF}$ & LDA & SVMrbf & $\mathrm{RF}$ & \\
\hline Cluster 1 & $83.59 \pm 0.19 \%$ & $99.19 \pm 0.22 \%$ & $99.37 \pm 0.56 \%$ & $84.26 \pm 0.26 \%$ & $99.41 \pm 0.07 \%$ & $99.56 \pm 0.00 \%$ & $\checkmark$ \\
\hline Cluster 2 & $94.30 \pm 1.48 \%$ & $99.93 \pm 0.00 \%$ & $99.85 \pm 0.00 \%$ & $94.93 \pm 2.19 \%$ & $99.73 \pm 0.07 \%$ & $99.76 \pm 0.04 \%$ & $\checkmark$ \\
\hline Cluster 3 & $95.33 \pm 1.48 \%$ & $99.93 \pm 0.07 \%$ & $99.93 \pm 0.07 \%$ & $95.48 \pm 1.78 \%$ & $99.85 \pm 0.07 \%$ & $99.85 \pm 0.07 \%$ & $\checkmark$ \\
\hline
\end{tabular}

$R F$ random forest; $S V M r b f$ support vector machine with radial basis function; $L D A$ linear discriminant analysis; $C V D$-CR cardiovascular diseasecurrent risk; $C V D$-3YFU cardiovascular disease risk-three-year follow-up

Table 13 Hypothesis: ML-based calculators are better than CCVRC calculators (using AUC score)

\begin{tabular}{lllllllll}
\hline Clusters & \multicolumn{2}{l}{ ML-based calculators } & & \multicolumn{2}{l}{ CCVRC calculators } & \multicolumn{2}{c}{ Hypothesis } \\
\cline { 2 - 3 } & LDA & SVMrbf & RF & & FRS & SCORE & ASCVD \\
\hline CVD-CR & 0.99 & 1.00 & 1.00 & 0.79 & 0.72 & 0.77 & $\checkmark$ \\
CVD-3YFU & 0.98 & 1.00 & 1.00 & 0.80 & 0.73 & 0.77 & $\checkmark$ \\
\hline
\end{tabular}

$R F$ random forest; $S V M r b f$ support vector machine with radial basis function; $L D A$ linear discriminant analysis; $C V D$ - $C R$ cardiovascular diseasecurrent risk; $C V D$-3YFU cardiovascular disease risk-three-year follow-up 
study, Jamthikar et al. [59] showed the use of an ML-based classifier called Random Forest for CVD risk prediction using 38 covariates that consisted of 13 conventional risk factors and 25 CUSIP ( 6 types of current CUSIP, 6 types of 10-year CUSIP, 12 types of quadratic CUSIP (harmonics), and age-adjusted grayscale median). Using RF classifier and Jack Knief (JK) protocol, the ML-based CVD risk assessment system showed an improvement of $(\sim 57 \%)$ against the conventional system $(\mathrm{AUC}=0.99, p$ value $<0.001$

Table 14 Benchmarking against ML-based CVD/stroke risk assessment in non-RA cohorts

\begin{tabular}{|c|c|c|c|c|c|c|c|c|}
\hline $\mathrm{C} 1$ & $\mathrm{C} 2$ & $\mathrm{C} 3$ & $\mathrm{C} 4$ & C6 & $\mathrm{C} 7$ & $\mathrm{C} 8$ & C9 & $\mathrm{C} 10$ \\
\hline $\mathrm{SN}$ & FA(Year) & Nop & FT & $\begin{array}{l}\text { Selected Fea- } \\
\text { tures }\end{array}$ & Classifiers & GS/GT Label & PE & $\begin{array}{l}\text { Benchmarking } \\
\text { Against }\end{array}$ \\
\hline $\mathrm{R} 1$ & $\begin{array}{r}\text { Gastounioti } \\
\text { et al. [61] }\end{array}$ & 56 & $\begin{array}{c}\text { Kinematics } \\
\text { Features }\end{array}$ & $\begin{array}{l}\text { FDR, PCA, } \\
\text { WRS }\end{array}$ & SVM & FU data labels & ACC: $88 \%$ & $\begin{array}{l}\text { DT, K-NN, DA, } \\
\text { PNN }\end{array}$ \\
\hline $\mathrm{R} 2$ & $\begin{array}{c}\text { Unnikrishnan } \\
\text { et al. [62] }\end{array}$ & 2406 & CCVR-F & NA & SVM & FU data labels & $\begin{array}{l}\text { Se: } 68.2 \%, \text { Sp: } \\
\text { 85.9\%, AUC: } \\
0.71\end{array}$ & FRS \\
\hline R3 & Weng et al. [63] & 378,256 & CCVR-F & - & $\begin{array}{l}\text { RFC, LR, GBM, } \\
\text { ANN }\end{array}$ & FU data labels & AUC: 0.764 & PCRS \\
\hline $\mathrm{R} 4$ & $\begin{array}{l}\text { Venkatesh et al. } \\
\text { [64] }\end{array}$ & 6814 & $\begin{array}{l}\text { CCVR-F, I-bf, } \\
\text { \& Serum } \\
\text { Biomarkers }\end{array}$ & MDMST & $\begin{array}{l}\text { RFC, Cox, } \\
\text { LASSO-cox, } \\
\text { AIC-Cox } \\
\text { backward } \\
\text { regression }\end{array}$ & FU data labels & $\begin{array}{c}\text { C-Index: } 0.81 \text {, } \\
\text { BS: } 0.083\end{array}$ & PCRS \& FRS \\
\hline R5 & Araki et al. [65] & 204 & I-bf & Statistical Test & SVM & $\begin{array}{l}\text { LD-based risk } \\
\text { labels }\end{array}$ & $\begin{array}{l}\text { ACC: NW: } \\
95.08 \%, \mathrm{FW}: \\
93.47 \%\end{array}$ & - \\
\hline R6 & $\begin{array}{l}\text { Banchhor et al. } \\
\text { [69] }\end{array}$ & 22 & $\begin{array}{l}\text { Texture \& wall- } \\
\text { based features }\end{array}$ & PCA & SVM & $\begin{array}{l}\text { Carotid plaque } \\
\text { burden }\end{array}$ & $\begin{array}{l}\text { ACC: } 91.28 \% \\
\text { AUC: } 0.91\end{array}$ & - \\
\hline R7 & $\begin{array}{l}\text { Kakadiaris et al. } \\
{[70]}\end{array}$ & 6459 & CCVR-F & - & SVM & FU data labels & $\begin{array}{l}\text { Se: } 86 \%, \text { Sp: } \\
\text { 95\%, AUC: } \\
0.92\end{array}$ & PCRS \\
\hline $\mathrm{R} 8$ & $\begin{array}{l}\text { Jamthikar et al. } \\
\text { [58] }\end{array}$ & 202 & $\begin{array}{l}\text { CCVR-F and } \\
\text { CUS I-bf }\end{array}$ & PCA polling & $\mathrm{RF}$ & $\begin{array}{l}\text { Carotid stenosis } \\
\text { Surrogate } \\
\text { endpoint of } \\
\text { CVD }\end{array}$ & $\begin{array}{l}\text { AUC of ML } \\
\text { system: } 0.80 \\
\text { (95\% CI: } \\
0.77-0.84) \\
\text { AUC for } \\
\text { CCVRC: } \\
0.68 \text { (95\% CI: } \\
0.64-0.72)\end{array}$ & - \\
\hline R9 & $\begin{array}{l}\text { Jamthikar et al. } \\
\text { [59] }\end{array}$ & 202 & $\begin{array}{l}\text { CCVR-F and } \\
\text { CUS I-bf }\end{array}$ & $\begin{array}{l}\text { Logistic Regres- } \\
\text { sion }\end{array}$ & $\mathrm{RF}$ & $\begin{array}{l}\text { LD as Surrogate } \\
\text { endpoint of } \\
\text { CVD }\end{array}$ & $\begin{array}{l}\text { AUC: } 0.99 \\
\quad(p<0.001)\end{array}$ & - \\
\hline R10 & $\begin{array}{l}\text { Jamthikar et al. } \\
\text { [60] }\end{array}$ & 202 & $\begin{array}{l}\text { CCVR-F and } \\
\text { CUS I-bf }\end{array}$ & - & SVM & $\begin{array}{l}\text { Surrogate end- } \\
\text { point of CVD }\end{array}$ & $\begin{array}{l}\text { AUC: } 0.88 \\
\quad(p<0.001)\end{array}$ & 13 CCVRC \\
\hline $\mathrm{R} 11$ & Proposed Study & 542 & $\begin{array}{l}\text { OBBM, LBBM, } \\
\text { CUSIP }\end{array}$ & $\begin{array}{l}\text { PCA pooling } \\
\quad \& \mathrm{MI}\end{array}$ & $\begin{array}{l}\text { RF, SVMrbf, } \\
\text { LDA }\end{array}$ & $\begin{array}{l}\text { CVE: (MI, or } \\
\text { stroke, or } \\
\text { Death), and } \\
\text { FU data labels }\end{array}$ & $\begin{array}{l}\text { ACC: } 98.40 \% \\
\text { for CVD- } \\
\text { CR and } \\
98.38 \% \text { for } \\
\text { CVD-3YFU, } \\
\text { AUC: } 0.96 \\
(p<0.0001)\end{array}$ & $\begin{array}{l}\text { CCVRC (FRS, } \\
\text { SCORE, and } \\
\text { ASCVD) }\end{array}$ \\
\hline
\end{tabular}

$S N$ serial num, Nop number of patients, $F U$ follow-up, $C V D$ cardiovascular disease, $C U S$ carotid ultrasound, $L D$ lumen diameter, $L R$ logistic regression, FDR Fisher discriminant ratio, WRS: Wilcoxon rank-sum, GS/GT labels: gold standard/GT labels, PCA: principal component analysis, MDMST: minimal depth of maximal subtree, SVMC support vector machine classifier, GMM: Gaussian mixture model, $D A$ discriminant analysis, RBPNN: Radial basis probabilistic neural network, I-bf: Image-based features, $D T$ Decision tree, $K$ - $N N$ K-Nearest Neighbors, $N B$ Naïve Bays, $F C$ Fuzzy Classifier, $F A$ first author, $Q N N$ : quantum neural network, $M L P$ : multilayer perceptron, $R F C$ : random forest classifier, $S O M$ self organization map, IGR: information gain ranking, $D B$ : database, $C C V R C$ : conventional cardiovascular risk calculators, ANN: artificial neural network, $D W T$ : discrete wavelet transform, $H o S$ higher-order spectra, $F T$ : features types, $C C V R$ - $F$ : conventional cardiovascular risk factors, $A C C$ : accuracy, $S e$ : sensitivity, $S p$ : specificity, $A U C$ : area under the curve, $B S$ Brier score, PCRS: pooled cohort risk score, and FRS: Framingham risk score, $C$-Index: Concordance index, $N W$ : near walls, $F W$ : far walls 
vs. $\mathrm{AUC}=0.63, p$ value $<0.001$, while considering 202 patients.

Jamthikar et al. [60], further showed the advantage of fusing carotid image-based phenotypes in ML framework using SVM. The authors compared their ML system against 13 types of conventional CVD risk calculators having the following performances (AECRS2.0 (AUC $0.83, p<0.001$ ), QRISK3 (AUC 0.72, $p<0.001$ ), WHO (AUC 0.70, $p<0.001$ ), ASCVD (AUC 0.67, $p<0.001$ ), FRS-cardio (AUC 0.67, $p<0.01$ ), FRS-stroke (AUC 0.64, $p<0.001$ ), MSRC (AUC 0.63, $p=0.03$ ), UKPDS56 (AUC $0.63, p<0.001$ ), NIPPON (AUC $0.63, p<0.001$ ), PROCAM (AUC 0.59, $p<0.001$ ), RRS (AUC 0.57, $p<0.001$ ), UKPDS60 (AUC 0.53, $p<0.001$ ), and SCORE (AUC 0.45, $p<0.001)$. The cohort consisted of 202 Japanese patients $(156 \mathrm{M} / 46 \mathrm{~F})$ having a mean age of $69 \pm 11$ years. Their system showed an improvement of $42 \%$ increase in performance having an AUC of $0.88(p<0.001)$. Gastounioti et al. [61] showed the advantage of kinematic features of the atherosclerotic plaque for 56 patients from two different hospitals with 15 computer-aided diagnoses (CAD) schemes in an ML-based framework with several cross-validation strategies. They used Fisher Discriminant Ratio (FDR), PCA, and Wilcoxon Rank-Sum (WRS) for selecting features with follow-up ground truth for the prediction of CVD risk assessment and they got $88 \%$ accuracy. Their methodology neither used CCVRC calculators, nor they had shown a feature effect. Further, the system did not consist of RA patients.

Unnikrishnan et al. [62] showed the study of cardiovascular risk assessment in 2406 participants with conventional cardiovascular risk factors in ML-based framework using Support Vector Machine classifier (SVM) classifier, benchmarking against Framingham risk score. Using SVM, the ML-based CVD risk assessment showed an AUC of $0.71,68.02 \%$ sensitivity (Se), and $85.9 \%$ specificity. Their methodology neither used feature selection technique nor had shown the effect of different clusters. Weng et al. [63] showed the improvement of cohort study for cardiovascular risk prediction in 378,256 patients from UK family practices using random forest classifier (RFC), logistic regression (LR), gradient boosting machines (GBM), benchmarking against pooled cohort risk score (PCRS), got an overall AUC of 0.764 . They neither used feature selection technique nor had benchmarking ML-based calculators against CCVRC. Further, their framework did not consist of RA patients.

Venkatesh et al. [64] predicted the six cardiovascular outcomes in comparison to standard cardiovascular risk scores and they had used 6814 test patients from the MESA (Multi-Ethnic Study of Atherosclerosis), aged between 45 and 84 years, from four different ethnicities, and six different centres across the United States. Using ML-based framework with Minimal Depth of Maximal Subtree as feature selection, random survival forests technique performed better than established risk scores with increased prediction accuracy, concordance index (C-Index) of 0.81, and Brier Score (BS) of 0.083. Araki et al. [65] showed the study of both the near and far walls of the carotid artery in Stroke risk assessment for 204 patients with Image-based features (I-bf) using Statistical Test as feature selection technique. Using SVM, they got an accuracy of $95.08 \%$ in near walls, $93.47 \%$ in far walls, respectively.

\section{A special note on SMOTE}

SMOTE [37, 66], also known as synthetic minority oversampling technique, is a well-known oversampling method where the synthetic samples were generated for the minority class. It is used for augmentation and for solving imbalance problems and is preferred in a generalized framework. While using SMOTE $5 \mathrm{X}$, there is an increase of performance accuracy for (i) LDA classifier from $86.53 \%$ to $95.33 \%$, (ii) SVMrbf classifier from $98.71 \%$ to $99.93 \%$, (iii) RF classifier from $98.71 \%$ to $99.93 \%$ for the current CVDCR setu The same behaviour was observed when using the CVD-3YFU setup, where the accuracy increased in (i) LDA classifier from $95.39 \%$ to $95.48 \%$, (ii) SVMrbf classifier increased from $98.52 \%$ to $99.85 \%$, and (iii) RF classifier increased from $98.52 \%$ to $99.85 \%$. This is because SMOTE creates the sample of the minority class, augment the dataset, and this technique helped to overcome the overfitting problem.

\section{A special note on CVD risk assessment during follow-up}

Even though the performance of classifiers in the CVD3 YFU set-up is equal to or better than CVD-CR, the number of CVE in both scenarios is very low. Only 7 out 542 had CVE in the CVD-CR setup, while 8 out of 542 had CVE in the CVD-3YFU setu This puts a strain on the ML classification system even if the SMOTE is activated. Note that in most of the clinical trials the CVE is typically $<5 \%$, however, in our case, this is $<1.5 \%$. This is one of the major weaknesses of our study. One solution would be to partner with other clinical centres which have a larger number of CVE. The second solution would be having a longer period of follow-up, however, this puts the constraint on the economics of healthcare. From the ML perspective, we successfully demonstrated that ML is stable and comparable in both scenarios (CVD-CR and CVD-3YFU) and easily extendable for larger period follow-up.

\section{Strengths/Weakness/Extensions}

ML-based CVD risk prediction is superior to CCVRC and can handle the non-linearity between the covariates, CVE 
in CVD-CR setup and CVD-3YFU setu This puts MLbased designs very effective and powerful when carotid ultrasound image-based phenotypes are integrated with the conventional and laboratory-based biomarkers. Further, SMOTE adds a powerful incentive when the sample size is small. The proposed system demonstrated a clear design where the future follow-ups CVD risks can be computed using the ML framework. While the four hypotheses were validated statistically, our dataset had limited cases of CVE cases during CVD-CR and CVD-3YFU setups. This was partially compensated by the SMOTE-based strategy and the SMOTE-based methodology was better in all the classification models. Since this is the first pilot study, there is a clear need for extensions to our models. A larger number of classification models can be tried, optimize the SMOTE paradigms, better cross-validation frameworks can be applied, and ensemble methods can be developed for the rheumatology framework. There is surely a potential of collecting multicenter RA and non-RA datasets to mixmatch the training model designs and even try the AI under unseen paradigms in big data framework [65]. Lastly, the machine learning models can be extended to deep learning frameworks $[67,68]$.

\section{Conclusions}

The proposed study is the first pilot study that used an MLbased system for CVD detection that used data at two different time points to establish associations between characteristics and current cardiovascular events or 3-year follow-up cardiovascular events. Three kinds of classifiers-Random Forest, Support Vector Machine, and Linear Discriminant Analysis were designed in SMOTE framework and compared against conventional cardiovascular disease CVD risk prediction calculators such as FRS, SCORE, and ASCVD. Our system demonstrated through-and-through the validation of the 4-hypothesis labelled as cluster-effect, SMOTEeffect, ML-effect, and follow-up effect. While using 46 covariates in 542 patients, the CVD-CR system showed the mean accuracy and AUC as $98.40 \%$ and $0.98(p<0.0001)$, while the CVD-3YFU showed the mean accuracy and ACU of $98.39 \%$ and $0.98(p<0.0001)$. The ML system was effective, clinically reliable, and reasonably fast (less than one second) during online prediction.

\section{Appendix A}

See Tables 15, 16, 17, 18, 19.
Table 15 Ordered list of covariates (selected feature) using PCA pooling

\begin{tabular}{|c|c|c|c|}
\hline \multicolumn{2}{|l|}{ CVD-CR } & \multicolumn{2}{|l|}{ CVD-3YFU } \\
\hline Covariates & $\begin{array}{l}\text { New SN (Old } \\
\text { SN) }\end{array}$ & Covariates & $\begin{array}{l}\text { New SN (Old } \\
\text { SN) }\end{array}$ \\
\hline LCB PLQ & $1(38)$ & $\mathrm{K}$ & $1(24)$ \\
\hline Height & $2(3)$ & $\mathrm{Tg}$ & $2(18)$ \\
\hline RICA PLQ & $3(42)$ & CPK & $3(30)$ \\
\hline CRP & $4(32)$ & RCCA PLQ & $4(40)$ \\
\hline $\mathrm{K}$ & $5(24)$ & LCB PLQ & $5(38)$ \\
\hline CPK & $6(30)$ & WBC & $6(21)$ \\
\hline RCCA PLQ & $7(40)$ & ESR & $7(23)$ \\
\hline $\mathrm{Tg}$ & $8(18)$ & HDL & $8(17)$ \\
\hline WBC & $9(21)$ & Height & $9(3)$ \\
\hline Gluc & $10(13)$ & RICA PLQ & $10(42)$ \\
\hline LICA PLQ & $11(39)$ & RCB PLQ & $11(41)$ \\
\hline $\mathrm{Na}$ & $12(25)$ & $\mathrm{Na}$ & $12(25)$ \\
\hline Urea & $13(27)$ & LCCA PLQ & $13(37)$ \\
\hline ESR & $14(23)$ & BMI & $14(4)$ \\
\hline PL & $15(22)$ & RCFA PLQ & $15(44)$ \\
\hline Diabetes T2D & $16(11)$ & LICA PLQ & $16(39)$ \\
\hline HR & $17(7)$ & Crp & $17(32)$ \\
\hline RCB PLQ & $18(41)$ & Age & $18(1)$ \\
\hline HDL & 19 (17) & Sex & $19(0)$ \\
\hline LCCA PLQ & $20(37)$ & Hypertension & $20(10)$ \\
\hline $\mathrm{Hg}$ & $21(20)$ & HR & $21(7)$ \\
\hline RCFA PLQ & $22(43)$ & PLT & $22(22)$ \\
\hline Create & $23(26)$ & $\mathrm{Hg}$ & $23(20)$ \\
\hline BMI & $24(4)$ & Gluc & $24(13)$ \\
\hline Age & $25(1)$ & HCT & 25 (19) \\
\hline AvDBP & $26(6)$ & Create & $26(26)$ \\
\hline HCT & 27 (19) & Urea & $27(27)$ \\
\hline TSH & $28(33)$ & Diabetes T2D & $28(11)$ \\
\hline Hypertension & $29(10)$ & LCFA PLQ & $29(43)$ \\
\hline UA & $30(31)$ & Alt & $30(29)$ \\
\hline AvSBP & $31(5)$ & AvDBP & $31(6)$ \\
\hline LCFA PLQ & $32(43)$ & UA & $32(31)$ \\
\hline Alt & 33 (29) & TSH & $33(33)$ \\
\hline Current Smoker & $34(9)$ & AST & $34(28)$ \\
\hline Sex & $35(0)$ & ${ }^{2} \mathrm{PLQ}$ & $35(34)$ \\
\hline Hyperlipidemia & $36(12)$ & ${ }^{1} \mathrm{FH}$ CAD & $36(8)$ \\
\hline AST & $37(28)$ & Weight & $37(2)$ \\
\hline${ }^{2} \mathrm{PLQ}$ & $38(34)$ & AvSBP & $38(5)$ \\
\hline${ }^{1} \mathrm{FH} \mathrm{CAD}$ & $39(8)$ & Current Smoker & $39(9)$ \\
\hline LDL & $40(16)$ & HbA1c & $40(14)$ \\
\hline $\mathrm{HbA1c}$ & $41(14)$ & Ldl & $41(16)$ \\
\hline${ }^{4} \mathrm{C}-\mathrm{PLQ}$ & $42(35)$ & Hyperlipidemia & $42(12)$ \\
\hline Weight & $43(2)$ & ${ }^{4} \mathrm{C}-\mathrm{PLQ}$ & $43(35)$ \\
\hline Chol & $44(15)$ & Chol & $44(15)$ \\
\hline${ }^{5}$ F-PLQ & $45(36)$ & ${ }^{5}$ F-PLQ & $45(36)$ \\
\hline${ }^{3}$ TNPLQ & $46(45)$ & ${ }^{3}$ TNPLQ & $46(45)$ \\
\hline
\end{tabular}


Table 15 (continued)

\section{$S N$ : Serial Number}

${ }^{1}$ FH CAD: family history of coronary artery disease

${ }^{2}$ PLQ: plaques $(\mathrm{Y} / \mathrm{N})$

${ }^{3}$ TNPLQ: total number of plaques

${ }^{4}$ C-PLQ: carotid plaque

${ }^{5}$ F-PLQ: femoral plaque

$A v S B P$ average systolic blood pressure; $A v \cdot D B P$ average diastolic blood pressure; $H R$ heart rate; $L C C A P L Q$ left common carotid artery plaque; $L C B P L Q$ left carotid bulb; $L I C A P L Q$ left internal carotid artery; $R C C A P L Q$ right common carotid artery plaque; $R C B P L Q$ right carotid bulb; RICA $P L Q$ right internal carotid artery; Gluc glucose; Chol cholesterol; $L D L$ low-density lipoprotein; $H D L$ highdensity lipoprotein; HbAlc hemoglobin A1c; PLT platelet count test; ESR erythrocyte sedimentation rate; $K$ potassium; $N a$ Sodium; $A S T$ aspartate aminotransferase; $A L T$ alanine aminotransferase; $C R P$ $\mathrm{C}$-reactive protein; $T S H$ thyroid-stimulating hormone; $U A$ uric acid

Table 16 Baseline characteristics for the rheumatology data for CVD-CR and CVD-3YFU

\begin{tabular}{|c|c|c|c|c|c|c|c|c|}
\hline \multirow[t]{2}{*}{ SN } & \multirow[t]{2}{*}{ Parameter } & \multirow[t]{2}{*}{ Overall (542) } & \multicolumn{3}{|c|}{ CVD-CR classes (No CVD and CVD) } & \multicolumn{3}{|c|}{ CVD-3YFU classes (No CVD and CVD) } \\
\hline & & & No CVD (535) & CVD (7) & $p$ value & No CVD (534) & CVD (8) & $p$ value \\
\hline \multicolumn{9}{|c|}{ Cluster 1: Baseline parameters (OBBM) } \\
\hline $\mathrm{R} 1$ & Sex (male) & $311(57.38 \%)$ & $307(98.71)$ & $4(1.29)$ & 0.831 & $306(98.39)$ & $5(1.61)$ & 0.934 \\
\hline $\mathrm{R} 2$ & Age (years) & $53.07 \pm 13.5$ & $52.99 \pm 13.5$ & $58.86 \pm 11.3$ & 0.253 & $53.00 \pm 13.5$ & $57.75 \pm 11.0$ & 0.323 \\
\hline R3 & Weight (kg) & $77.27 \pm 17.0$ & $77.26 \pm 17.1$ & $78.04 \pm 14.4$ & 0.904 & $77.24 \pm 17.1$ & $79.47 \pm 14.0$ & 0.713 \\
\hline $\mathrm{R} 4$ & Height (meter) & $1.65 \pm 0.1$ & $1.65 \pm 0.1$ & $1.63 \pm 0.1$ & 0.568 & $1.65 \pm 0.1$ & $1.63 \pm 0.1$ & 0.538 \\
\hline R5 & $\operatorname{BMI}\left(\mathrm{kg} / \mathrm{m}^{2}\right)$ & $28.17 \pm 5.3$ & $28.16 \pm 5.3$ & $29.14 \pm 4.3$ & 0.626 & $28.15 \pm 5.3$ & $29.71 \pm 4.3$ & 0.406 \\
\hline R6 & $\mathrm{AvSBP}(\mathrm{mmHg})$ & $130.61 \pm 19.1$ & $130.60 \pm 19.2$ & $130.86 \pm 14.4$ & 0.972 & $130.60 \pm 19.2$ & $130.62 \pm 13.5$ & 0.998 \\
\hline $\mathrm{R} 7$ & AvDBP (mmHg) & $78.44 \pm 11.0$ & $78.44 \pm 11.1$ & $78.86 \pm 8.1$ & 0.920 & $78.43 \pm 11.1$ & $79.25 \pm 7.7$ & 0.835 \\
\hline $\mathrm{R} 8$ & HR (bpm) & $68.31 \pm 10.5$ & $68.37 \pm 10.4$ & $64.14 \pm 11.7$ & 0.290 & $68.38 \pm 10.5$ & $63.75 \pm 11.0$ & 0.215 \\
\hline R9 & ${ }^{1} \mathrm{FH} \mathrm{CAD}(\%)$ & $72(13.3 \%)$ & $71(98.6 \%)$ & $1(1.4 \%)$ & 0.630 & $70(97.2 \%)$ & $2(2.8 \%)$ & 0.646 \\
\hline $\mathrm{R} 10$ & Current Smoker ${ }^{\dagger}$ & $0.76 \pm 0.8$ & $0.75 \pm 0.8$ & $1.57 \pm 0.7$ & 0.006 & $0.75 \pm 0.8$ & $1.50 \pm 0.7$ & 0.007 \\
\hline $\mathrm{R} 11$ & Hypertension $(\mathrm{mmHg})$ & $203(37.5 \%)$ & $198(97.5 \%)$ & $5(2.5 \%)$ & 0.140 & 197(97.0\%) & $6(3.0 \%)$ & 0.065 \\
\hline $\mathrm{R} 12$ & Diabetes T2D (mg/dl) & $0.36 \pm 0.7$ & $0.36 \pm 0.7$ & $0.29 \pm 0.5$ & 0.785 & $0.36 \pm 0.7$ & $0.25 \pm 0.4$ & 0.663 \\
\hline $\mathrm{R} 13$ & Hyperlipidemia (\%) & $147(27.1 \%)$ & $143(97.3 \%)$ & $4(2.7 \%)$ & 0.171 & $142(96.6 \%)$ & $5(3.4 \%)$ & 0.062 \\
\hline \multicolumn{9}{|c|}{ Cluster 2: OBBM+LBBM } \\
\hline $\mathrm{R} 14$ & Gluc (mmol/l) & $102.61 \pm 31.5$ & $102.55 \pm 31.6$ & $107.14 \pm 28.2$ & 0.703 & $102.58 \pm 31.6$ & $104.75 \pm 27.2$ & 0.847 \\
\hline $\mathrm{R} 15$ & $\mathrm{HbA} 1 \mathrm{c}(\mathrm{mg} / \mathrm{dl})$ & $5.94 \pm 0.9$ & $5.93 \pm 0.9$ & $6.23 \pm 0.7$ & 0.372 & $5.93 \pm 0.9$ & $6.14 \pm 0.7$ & 0.511 \\
\hline $\mathrm{R} 16$ & $\mathrm{Chol}^{+}(\mathrm{mg} / \mathrm{dl})$ & $202.93 \pm 37.9$ & $203.36 \pm 37.6$ & $170.57 \pm 46.7$ & $\mathbf{0 . 0 2 3}$ & $203.44 \pm 37.6$ & $168.88 \pm 43.9$ & 0.010 \\
\hline $\mathrm{R} 17$ & $\mathrm{LDL}^{+}(\mathrm{mg} / \mathrm{dl})$ & $125.02 \pm 32.1$ & $125.37 \pm 31.9$ & $98.14 \pm 34.9$ & 0.026 & $125.42 \pm 31.9$ & $98.25 \pm 32.7$ & 0.017 \\
\hline $\mathrm{R} 18$ & HDL (mg/dl) & $57.23 \pm 15.2$ & $57.30 \pm 15.2$ & $52.29 \pm 16.6$ & 0.386 & $57.33 \pm 15.1$ & $50.75 \pm 16.1$ & 0.224 \\
\hline R19 & $\operatorname{Tg}(\mathrm{mg} / \mathrm{dl})$ & $110.10 \pm 57.2$ & $110.17 \pm 57.4$ & $104.71 \pm 39.9$ & 0.802 & $110.21 \pm 57.4$ & $102.62 \pm 37.7$ & 0.710 \\
\hline $\mathrm{R} 20$ & $\operatorname{Hct}(\mathrm{mg} / \mathrm{dl})$ & $41.27 \pm 3.5$ & $41.29 \pm 3.5$ & $39.84 \pm 2.4$ & 0.285 & $41.29 \pm 3.6$ & $39.92 \pm 2.2$ & 0.281 \\
\hline $\mathrm{R} 21$ & $\mathrm{Hg}(\mathrm{mg} / \mathrm{dl})$ & $13.69 \pm 1.7$ & $13.70 \pm 1.7$ & $12.89 \pm 1.0$ & 0.220 & $13.70 \pm 1.7$ & $12.99 \pm 1.0$ & 0.252 \\
\hline $\mathrm{R} 22$ & WBC (K/ul) & $6836.75 \pm 1766.9$ & $6838.22 \pm 1777.5$ & $6724.29 \pm 483.4$ & 0.866 & $6830.06 \pm 1769.1$ & $7283.75 \pm 1547.7$ & 0.472 \\
\hline $\mathrm{R} 23$ & PLT (mg/dl) & $248.89 \pm 68.0$ & $248.69 \pm 68.0$ & $264.29 \pm 69.4$ & 0.548 & $248.82 \pm 68.0$ & $253.62 \pm 70.8$ & 0.843 \\
\hline $\mathrm{R} 24$ & $\mathrm{ESR}(\mathrm{mm} / \mathrm{hr})$ & $15.46 \pm 13.1$ & $15.34 \pm 12.6$ & $24.71 \pm 33.8$ & 0.060 & $15.35 \pm 12.6$ & $23.00 \pm 31.9$ & 0.102 \\
\hline $\mathrm{R} 25$ & $\mathrm{~K}(\mathrm{mEq} / \mathrm{l})$ & $4.38 \pm 0.3$ & $4.38 \pm 0.3$ & $4.31 \pm 0.2$ & 0.595 & $4.38 \pm 0.3$ & $4.39 \pm 0.2$ & 0.939 \\
\hline $\mathrm{R} 26$ & $\mathrm{Na}(\mathrm{mEq} / \mathrm{l})$ & $140.70 \pm 2.0$ & $140.70 \pm 2.0$ & $141.10 \pm 1.7$ & 0.592 & $140.69 \pm 2.0$ & $141.34 \pm 1.7$ & 0.360 \\
\hline $\mathrm{R} 27$ & Create $(\mathrm{mEq} / \mathrm{l})$ & $0.85 \pm 0.2$ & $0.85 \pm 0.2$ & $0.90 \pm 0.2$ & 0.536 & $0.85 \pm 0.2$ & $0.89 \pm 0.2$ & 0.631 \\
\hline $\mathrm{R} 28$ & Urea (mg/dl) & $33.36 \pm 9.4$ & $33.30 \pm 9.4$ & $37.93 \pm 9.7$ & 0.194 & $33.29 \pm 9.4$ & $37.44 \pm 9.2$ & 0.215 \\
\hline $\mathrm{R} 29$ & $\operatorname{AST}(\mathrm{u} / \mathrm{l})$ & $21.25 \pm 7.9$ & $21.23 \pm 7.9$ & $22.61 \pm 3.0$ & 0.646 & $21.25 \pm 7.9$ & $21.29 \pm 4.5$ & 0.990 \\
\hline
\end{tabular}


Table 16 (continued)

\begin{tabular}{|c|c|c|c|c|c|c|c|c|}
\hline \multirow[t]{2}{*}{ SN } & \multirow[t]{2}{*}{ Parameter } & \multirow[t]{2}{*}{ Overall (542) } & \multicolumn{3}{|c|}{ CVD-CR classes (No CVD and CVD) } & \multicolumn{3}{|c|}{ CVD-3YFU classes (No CVD and CVD) } \\
\hline & & & No CVD (535) & CVD (7) & $p$ value & No CVD (534) & CVD (8) & $p$ value \\
\hline R30 & $\operatorname{ALT}(\mathrm{u} / \mathrm{l})$ & $24.51 \pm 14.5$ & $24.54 \pm 14.6$ & $22.71 \pm 4.1$ & 0.742 & $24.55 \pm 14.6$ & $21.75 \pm 4.6$ & 0.589 \\
\hline $\mathrm{R} 31$ & $\mathrm{CPK}(\mathrm{mg} / \mathrm{l})$ & $95.62 \pm 67.7$ & $95.94 \pm 68.1$ & $71.07 \pm 13.4$ & 0.335 & $95.96 \pm 68.1$ & $73.19 \pm 13.8$ & 0.346 \\
\hline $\mathrm{R} 32$ & $\mathrm{UA}^{+}(\mathrm{mg} / \mathrm{l})$ & $4.60 \pm 1.2$ & $4.61 \pm 1.2$ & $3.62 \pm 0.3$ & $\mathbf{0 . 0 3 3}$ & $4.61 \pm 1.2$ & $3.72 \pm 0.4$ & 0.040 \\
\hline $\mathrm{R} 33$ & $\mathrm{CRP}^{+}(\mathrm{mg} / \mathrm{l})$ & $4.27 \pm 5.5$ & $4.20 \pm 5.4$ & $9.19 \pm 11.4$ & 0.018 & $4.19 \pm 5.4$ & $9.41 \pm 10.7$ & 0.008 \\
\hline $\mathrm{R} 34$ & TSH (mlU/l) & $1.62 \pm 0.7$ & $1.61 \pm 0.7$ & $1.78 \pm 0.3$ & 0.558 & $1.61 \pm 0.7$ & $2.27 \pm 1.3$ & 0.013 \\
\hline \multicolumn{9}{|c|}{ Cluster 3: OBBM + LBBM + CUSIP } \\
\hline R35 & ${ }^{2} \mathrm{PLQ}\left(\mathrm{mm}^{2}\right)$ & $260(48.0 \%)$ & $255(98.1 \%)$ & $5(1.9 \%)$ & 0.384 & $254(97.7 \%)$ & $6(2.3 \%)$ & 0.236 \\
\hline $\mathrm{R} 36$ & ${ }^{4} \mathrm{C}-\mathrm{PLQ}\left(\mathrm{mm}^{2}\right)$ & $187(34.5 \%)$ & $183(97.9 \%)$ & $4(2.1 \%)$ & 0.385 & $182(97.3 \%)$ & $5(2.7 \%)$ & 0.192 \\
\hline R37 & ${ }^{5} \mathrm{~F}-\mathrm{PLQ}\left(\mathrm{mm}^{2}\right)$ & $190(35.1 \%)$ & $187(98.4 \%)$ & $3(1.6 \%)$ & 0.971 & $186(97.9 \%)$ & $4(2.1 \%)$ & 0.604 \\
\hline R38 & LCCA PLQ $\left(\mathrm{mm}^{2}\right)$ & $15(2.8 \%)$ & $15(100.0 \%)$ & $0(0.0 \%)$ & 0.478 & $15(100.0 \%)$ & $0(0.0 \%)$ & 0.545 \\
\hline $\mathrm{R} 39$ & $\operatorname{LCB}$ PLQ $\left(\mathrm{mm}^{2}\right)$ & $119(22.0 \%)$ & $117(98.3 \%)$ & $2(1.7 \%)$ & 0.973 & $117(98.3 \%)$ & $2(1.7 \%)$ & 0.825 \\
\hline $\mathrm{R} 40$ & LICA PLQ $\left(\mathrm{mm}^{2}\right)$ & $39(7.2 \%)$ & $39(100.0 \%)$ & $0(0.0 \%)$ & 0.996 & $38(97.4 \%)$ & $1(2.6 \%)$ & 0.917 \\
\hline $\mathrm{R} 41$ & RCCA PLQ $\left(\mathrm{mm}^{2}\right)$ & $11(2.0 \%)$ & $11(100.0 \%)$ & $0(0.0 \%)$ & 0.334 & $11(100.0 \%)$ & $0(0.0 \%)$ & 0.394 \\
\hline $\mathrm{R} 42$ & RCB PLQ $\left(\mathrm{mm}^{2}\right)$ & $132(24.4 \%)$ & $129(97.7 \%)$ & $3(2.3 \%)$ & 0.481 & $128(97.0 \%)$ & $4(3.0 \%)$ & 0.198 \\
\hline $\mathrm{R} 43$ & RICA PLQ $\left(\mathrm{mm}^{2}\right)$ & $25(4.6 \%)$ & $25(100.0 \%)$ & $0(0.0 \%)$ & 0.748 & $25(100.0 \%)$ & $0(0.0 \%)$ & 0.824 \\
\hline R44 & LCFA PLQ $\left(\mathrm{mm}^{2}\right)$ & $139(25.6 \%)$ & $137(98.6 \%)$ & $2(1.4 \%)$ & 0.797 & $136(97.8 \%)$ & $3(2.2 \%)$ & 0.715 \\
\hline $\mathrm{R} 45$ & RCFA PLQ $\left(\mathrm{mm}^{2}\right)$ & $164(30.3 \%)$ & $162(98.8 \%)$ & $2(1.2 \%)$ & 0.752 & $161(98.2 \%)$ & $3(1.8 \%)$ & 0.951 \\
\hline $\mathrm{R} 46$ & ${ }^{3} \mathrm{TNPLQ}\left(\mathrm{mm}^{2}\right)$ & $1.19 \pm 1.6$ & $1.19 \pm 1.6$ & $1.29 \pm 0.9$ & 0.867 & $1.18 \pm 1.6$ & $1.62 \pm 1.2$ & 0.429 \\
\hline
\end{tabular}

+ Significant cutoff of risk covariate is $p<0.05$

${ }^{1} \mathrm{FH} C A D$ family history of coronary artery disease

${ }^{2} P L Q$ plaques $(\mathrm{Y} / \mathrm{N})$

${ }^{3} T N P L Q$ total number of plaques

${ }^{4} C$ - $P L Q$ : carotid plaque

${ }^{5} F-P L Q$ : femoral plaque

$A v S B P$ : average systolic blood pressure; $A v . D B P$ : average diastolic blood pressure; $H R$ : heart rate; $L C C A P L Q$ : left common carotid artery plaque; $L C B P L Q$ : left carotid bulb; $L I C A P L Q$ : left internal carotid artery; $R C C A P L Q$ : right common carotid artery plaque; $R C B P L Q$ right carotid bulb; HbAlc hemoglobin A1C; RICA PLQ right internal carotid artery; Gluc glucose; Chol: cholesterol; $L D L$ low-density lipoprotein; $H D L$ high-density lipoprotein; $T g$ triglyceride; Hct hematocrit; HbAlc hemoglobin A1c; PLT platelet count test; ESR erythrocyte sedimentation rate; $K$ potassium; $N a$ Sodium; $A S T$ aspartate aminotransferase; $A L T$ alanine aminotransferase; $C R P$ C-reactive protein; $U A$ uric acid

Table 17 Accuracy comparison MI (with top 50\% MI scores) vs. PCA pooling

\begin{tabular}{llllllll}
\hline Classifiers & \multicolumn{2}{l}{ CVD-CR } & \multicolumn{5}{l}{ CVD-3YFU } \\
\cline { 2 - 3 } \cline { 7 - 8 } & No FS & MI & PCA pooling & & No FS & MI & PCA pooling \\
\hline RF & $97.26 \%$ & $98.53 \%$ & $98.71 \%$ & & $98.53 \%$ & $98.53 \%$ & $98.52 \%$ \\
SVMrbf & $95.59 \%$ & $96.32 \%$ & $98.71 \%$ & & $97.06 \%$ & $98.53 \%$ & $98.52 \%$ \\
LDA & $95.59 \%$ & $96.32 \%$ & $96.53 \%$ & & $94.85 \%$ & $94.96 \%$ & $95.39 \%$ \\
Mean & $96.15 \%$ & $97.06 \%$ & $97.98 \%$ & & $96.81 \%$ & $97.34 \%$ & $97.48 \%$ \\
SD & 0.01 & 0.01 & 0.001 & & 0.02 & 0.02 & 0.02 \\
\hline
\end{tabular}

$F S$ feature selection, $R F$ random forest; $S V M r b f$ support vector machine with radial basis function; $L D A$ linear discriminant analysis; $C V D$ - $C R$ cardiovascular disease-current risk; $C V D-3 Y F U$ cardiovascular disease risk-three-year follow-up 
Table 18 Shapiro Test $\mathrm{p}$ values for 46 covariates in CVD-CR and CVD-3YFU frameworks

\begin{tabular}{|c|c|c|c|c|c|c|c|}
\hline \multirow[t]{2}{*}{$\mathrm{SN}$} & \multirow[t]{2}{*}{ Covariates } & \multicolumn{3}{|l|}{ CVD-CR } & \multicolumn{3}{|l|}{ CVD-3YFU } \\
\hline & & Overall & No CVD & CVD & Overall & No CVD & CVD \\
\hline $\mathrm{R} 1$ & Sex & $<0.0001$ & $<0.0001$ & $<0.0001$ & $<0.0001$ & $<0.0001$ & $<0.0001$ \\
\hline R2 & Age & $<0.0001$ & $<0.0001$ & 0.400 & $<0.0001$ & $<0.0001$ & 0.700 \\
\hline R3 & Weight & $<0.0001$ & $<0.0001$ & $<0.0001$ & $<0.0001$ & $<0.0001$ & $<0.0001$ \\
\hline R4 & Height & $<0.0001$ & $<0.0001$ & 0.500 & $<0.0001$ & $<0.0001$ & 0.400 \\
\hline R5 & BMI & $<0.0001$ & $<0.0001$ & $<0.0001$ & $<0.0001$ & $<0.0001$ & $<0.0001$ \\
\hline R6 & AvSBP & $<0.0001$ & $<0.0001$ & 0.200 & $<0.0001$ & $<0.0001$ & 0.200 \\
\hline $\mathrm{R} 7$ & AvDBP & $<0.0001$ & $<0.0001$ & 0.200 & $<0.0001$ & $<0.0001$ & 0.300 \\
\hline R8 & HR & $<0.0001$ & $<0.0001$ & 0.300 & $<0.0001$ & $<0.0001$ & 0.200 \\
\hline R9 & ${ }^{1} \mathrm{FH} \mathrm{CAD}$ & $<0.0001$ & $<0.0001$ & $<0.0001$ & $<0.0001$ & $<0.0001$ & $<0.0001$ \\
\hline $\mathrm{R} 10$ & Current Smoker & $<0.0001$ & $<0.0001$ & $<0.0001$ & $<0.0001$ & $<0.0001$ & $<0.0001$ \\
\hline R11 & Hypertension & $<0.0001$ & $<0.0001$ & $<0.0001$ & $<0.0001$ & $<0.0001$ & $<0.0001$ \\
\hline R12 & Diabetes T2D & $<0.0001$ & $<0.0001$ & $<0.0001$ & $<0.0001$ & $<0.0001$ & $<0.0001$ \\
\hline $\mathrm{R} 13$ & Hyperlipidemia & $<0.0001$ & $<0.0001$ & $<0.0001$ & $<0.0001$ & $<0.0001$ & $<0.0001$ \\
\hline R14 & Gluc & $<0.0001$ & $<0.0001$ & $<0.0001$ & $<0.0001$ & $<0.0001$ & $<0.0001$ \\
\hline R15 & $\mathrm{HbA1c}$ & $<0.0001$ & $<0.0001$ & $<0.0001$ & $<0.0001$ & $<0.0001$ & $<0.0001$ \\
\hline R16 & Chol & $<0.0001$ & $<0.0001$ & $<0.0001$ & $<0.0001$ & $<0.0001$ & $<0.0001$ \\
\hline R17 & LDL & $<0.0001$ & $<0.0001$ & 0.300 & $<0.0001$ & $<0.0001$ & 0.200 \\
\hline $\mathrm{R} 18$ & HDL & $<0.0001$ & $<0.0001$ & 0.900 & $<0.0001$ & $<0.0001$ & 0.900 \\
\hline R19 & $\mathrm{Tg}$ & $<0.0001$ & $<0.0001$ & 0.900 & $<0.0001$ & $<0.0001$ & 0.900 \\
\hline $\mathrm{R} 20$ & HCT & $<0.0001$ & $<0.0001$ & 0.500 & $<0.0001$ & $<0.0001$ & 0.400 \\
\hline $\mathrm{R} 21$ & $\mathrm{Hg}$ & $<0.0001$ & $<0.0001$ & 0.500 & $<0.0001$ & $<0.0001$ & 0.900 \\
\hline $\mathrm{R} 22$ & WBC & $<0.0001$ & $<0.0001$ & 0.200 & $<0.0001$ & $<0.0001$ & $<0.0001$ \\
\hline $\mathrm{R} 23$ & PLT & $<0.0001$ & $<0.0001$ & 0.100 & $<0.0001$ & $<0.0001$ & 0.100 \\
\hline R24 & ESR & $<0.0001$ & $<0.0001$ & $<0.0001$ & $<0.0001$ & $<0.0001$ & $<0.0001$ \\
\hline $\mathrm{R} 25$ & K & $<0.0001$ & $<0.0001$ & 0.100 & $<0.0001$ & $<0.0001$ & 0.200 \\
\hline R26 & $\mathrm{Na}$ & $<0.0001$ & $<0.0001$ & 0.300 & $<0.0001$ & $<0.0001$ & 0.200 \\
\hline R27 & Create & $<0.0001$ & $<0.0001$ & 0.100 & $<0.0001$ & $<0.0001$ & $<0.0001$ \\
\hline $\mathrm{R} 28$ & Urea & $<0.0001$ & $<0.0001$ & $<0.0001$ & $<0.0001$ & $<0.0001$ & $<0.0001$ \\
\hline R29 & AST & $<0.0001$ & $<0.0001$ & 0.200 & $<0.0001$ & $<0.0001$ & 0.500 \\
\hline R30 & ALT & $<0.0001$ & $<0.0001$ & 0.800 & $<0.0001$ & $<0.0001$ & 0.500 \\
\hline R31 & CPK & $<0.0001$ & $<0.0001$ & $<0.0001$ & $<0.0001$ & $<0.0001$ & $<0.0001$ \\
\hline R32 & UA & $<0.0001$ & $<0.0001$ & $<0.0001$ & $<0.0001$ & $<0.0001$ & $<0.0001$ \\
\hline R33 & CRP & $<0.0001$ & $<0.0001$ & $<0.0001$ & $<0.0001$ & $<0.0001$ & $<0.0001$ \\
\hline R34 & TSH & $<0.0001$ & $<0.0001$ & $<0.0001$ & $<0.0001$ & $<0.0001$ & $<0.0001$ \\
\hline R35 & ${ }^{2} \mathrm{PLQ}$ & $<0.0001$ & $<0.0001$ & $<0.0001$ & $<0.0001$ & $<0.0001$ & $<0.0001$ \\
\hline R36 & ${ }^{4} \mathrm{C}-\mathrm{PLQ}$ & $<0.0001$ & $<0.0001$ & $<0.0001$ & $<0.0001$ & $<0.0001$ & $<0.0001$ \\
\hline R37 & ${ }^{5} \mathrm{~F}-\mathrm{PLQ}$ & $<0.0001$ & $<0.0001$ & $<0.0001$ & $<0.0001$ & $<0.0001$ & $<0.0001$ \\
\hline R38 & LCCA PLQ & $<0.0001$ & $<0.0001$ & 1.000 & $<0.0001$ & $<0.0001$ & 1.000 \\
\hline R39 & LCB PLQ & $<0.0001$ & $<0.0001$ & $<0.0001$ & $<0.0001$ & $<0.0001$ & $<0.0001$ \\
\hline R40 & LICA PLQ & $<0.0001$ & $<0.0001$ & 1.000 & $<0.0001$ & $<0.0001$ & $<0.0001$ \\
\hline R41 & RCCA PLQ & $<0.0001$ & $<0.0001$ & 1.000 & $<0.0001$ & $<0.0001$ & 1.000 \\
\hline R42 & RCB PLQ & $<0.0001$ & $<0.0001$ & $<0.0001$ & $<0.0001$ & $<0.0001$ & $<0.0001$ \\
\hline R43 & RICA PLQ & $<0.0001$ & $<0.0001$ & 1.000 & $<0.0001$ & $<0.0001$ & 1.000 \\
\hline R44 & LCFA PLQ & $<0.0001$ & $<0.0001$ & $<0.0001$ & $<0.0001$ & $<0.0001$ & $<0.0001$ \\
\hline R45 & RCFA PLQ & $<0.0001$ & $<0.0001$ & $<0.0001$ & $<0.0001$ & $<0.0001$ & $<0.0001$ \\
\hline R46 & ${ }^{3}$ TNPLQ & $<0.0001$ & $<0.0001$ & $<0.0001$ & $<0.0001$ & $<0.0001$ & 0.200 \\
\hline
\end{tabular}

${ }^{1} F H C A D$ : family history of coronary artery disease

${ }^{2} P L Q$ : plaques $(\mathrm{Y} / \mathrm{N})$

${ }^{3} T N P L Q$ : total number of plaques; ${ }^{4} C-P L Q$ carotid plaque; ${ }^{5} F-P L Q$ femoral plaque; $A v S B P$ average systolic blood pressure; $A v . D B P$ average diastolic blood pressure; $H R$ heart rate; $L C C A P L Q$ left common carotid artery Plaque; $L C B P L Q$ left carotid bulb; $L I C A P L Q$ left internal carotid artery; $R C C A P L Q$ right common carotid artery plaque; $R C B P L Q$ right carotid bulb; RICA PLQ right internal carotid artery; Gluc glucose; Chol cholesterol; $L D L$ low-density lipoprotein; $H D L$ high-density lipoprotein; HbAlc hemoglobin A1c; PLT platelet count test; ESR erythrocyte sedimentation rate; $K$ potassium; $N a$ Sodium; AST: aspartate aminotransferase; $A L T$ alanine aminotransferase; $C R P$ C-reactive protein; TSH thyroid-stimulating hormone; UA: uric acid 
Table 19 Baseline characteristics for the validation coronary database

\begin{tabular}{|c|c|c|c|c|c|}
\hline \multirow[t]{2}{*}{$\mathrm{SN}$} & \multirow[t]{2}{*}{ Parameter } & \multirow[t]{2}{*}{ Overall } & \multicolumn{2}{|c|}{ AngioScore classes ( 2 class) } & \multirow[t]{2}{*}{$p$ value } \\
\hline & & & No-Risk & High Risk & \\
\hline $\mathrm{R} 1$ & $\mathrm{Age}^{+}$ & $64.49 \pm 10.5$ & $62.76 \pm 10.9$ & $65.67 \pm 10.1$ & 0.002 \\
\hline R2 & Caucasian & $486(97.2 \%)$ & $195(40.1 \%)$ & $291(59.9 \%)$ & 0.641 \\
\hline R3 & ${ }^{1} \mathrm{ADba}$ & $76.74 \pm 13.7$ & $76.84 \pm 14.0$ & $76.67 \pm 13.5$ & 0.897 \\
\hline R4 & Pre-Diabetic & $20(4.0 \%)$ & $7(35.0 \%)$ & $13(65.0 \%)$ & 0.787 \\
\hline R5 & Diabetes T1D & $5(1.0 \%)$ & $3(60.0 \%)$ & $2(40.0 \%)$ & 0.660 \\
\hline R6 & Diabetes $\mathrm{T}_{2} \mathrm{D}^{+}$ & $114(22.8 \%)$ & $33(28.9 \%)$ & $81(71.1 \%)$ & 0.006 \\
\hline R7 & Diabetes (any) ${ }^{+}$ & $118(23.6 \%)$ & $36(30.5 \%)$ & $82(69.5 \%)$ & 0.016 \\
\hline R8 & Hypertension & $338(67.6 \%)$ & $126(37.3 \%)$ & $212(62.7 \%)$ & 0.050 \\
\hline R9 & Hyperlipidemia ${ }^{\dagger}$ & $288(57.6 \%)$ & $98(34.0 \%)$ & $190(66.0 \%)$ & 0.001 \\
\hline $\mathrm{R} 10$ & ${ }^{2} \mathrm{ASba}$ & $135.35 \pm 21.4$ & $135.01 \pm 20.9$ & $135.59 \pm 21.8$ & 0.767 \\
\hline $\mathrm{R} 11$ & Current Smoker ${ }^{\dagger}$ & $100(20.0 \%)$ & $29(29.0 \%)$ & $71(71.0 \%)$ & 0.013 \\
\hline $\mathrm{R} 12$ & Casual Smoker & $15(3.0 \%)$ & $5(33.3 \%)$ & $10(66.7 \%)$ & 0.765 \\
\hline $\mathrm{R} 13$ & Previous Smoker & $218(43.6 \%)$ & $87(39.9 \%)$ & $131(60.1 \%)$ & 0.916 \\
\hline $\mathrm{R} 14$ & Smoking $\mathrm{Hx}^{\dagger}$ & $330(66.0 \%)$ & $120(36.4 \%)$ & $210(63.6 \%)$ & 0.014 \\
\hline $\mathrm{R} 15$ & $\operatorname{Sex}^{+}$ & $349(69.8 \%)$ & $119(34.1 \%)$ & $230(65.9 \%)$ & $<0.0001$ \\
\hline R16 & $\mathrm{GFR}^{+}$ & $78.96 \pm 18.2$ & $81.02 \pm 17.6$ & $77.57 \pm 18.4$ & 0.037 \\
\hline $\mathrm{R} 17$ & Creatinine $^{+}$ & $83.99 \pm 22.6$ & $80.89 \pm 20.9$ & $86.10 \pm 23.4$ & 0.011 \\
\hline R18 & Obesity & $215(43.0 \%)$ & $98(45.6 \%)$ & $117(54.4 \%)$ & 0.050 \\
\hline R19 & BMI & $30.41 \pm 6.3$ & $30.82 \pm 6.6$ & $30.14 \pm 6.0$ & 0.228 \\
\hline $\mathrm{R} 20$ & ${ }^{3}$ FHPCVD & $146(29.2 \%)$ & $62(42.5 \%)$ & $84(57.5 \%)$ & 0.614 \\
\hline $\mathrm{R} 21$ & ${ }^{4} \mathrm{FHD}$ & $195(39.0 \%)$ & $76(39.0 \%)$ & $119(61.0 \%)$ & 0.670 \\
\hline $\mathrm{R} 22$ & Drinks/wk & $4.86 \pm 10.4$ & $4.64 \pm 9.2$ & $5.00 \pm 11.1$ & 0.706 \\
\hline R23 & Angina & $124(24.8 \%)$ & $47(37.9 \%)$ & $77(62.1 \%)$ & 0.584 \\
\hline R24 & Family Hx of CVD & $321(64.2 \%)$ & $123(38.3 \%)$ & $198(61.7 \%)$ & 0.240 \\
\hline $\mathrm{R} 25$ & ${ }^{5} \mathrm{HCRI}^{\dagger}$ & $272(54.4 \%)$ & $94(34.6 \%)$ & $178(65.4 \%)$ & 0.005 \\
\hline $\mathrm{R} 26$ & ${ }^{6} \mathrm{OAA}$ & $9(1.8 \%)$ & $4(44.4 \%)$ & $5(55.6 \%)$ & 0.926 \\
\hline R27 & ACE Inhibitors & $191(38.2 \%)$ & $72(37.7 \%)$ & $119(62.3 \%)$ & 0.382 \\
\hline $\mathrm{R} 28$ & ARBs Angiotensis & $45(9.0 \%)$ & $13(28.9 \%)$ & $32(71.1 \%)$ & 0.136 \\
\hline R29 & Alpha-Blockers & $30(6.0 \%)$ & $10(33.3 \%)$ & $20(66.7 \%)$ & 0.534 \\
\hline $\mathrm{R} 30$ & Beta-Blockers' & $236(47.2 \%)$ & $77(32.6 \%)$ & $159(67.4 \%)$ & 0.001 \\
\hline $\mathrm{R} 31$ & ${ }^{7} \mathrm{CCB}$ & $93(18.6 \%)$ & $37(39.8 \%)$ & $56(60.2 \%)$ & 0.987 \\
\hline $\mathrm{R} 32$ & ${ }^{8} \mathrm{AP} / \mathrm{AC}^{+}$ & $368(73.6 \%)$ & $131(35.6 \%)$ & $237(64.4 \%)$ & $<0.0001$ \\
\hline R33 & Diuretics & $99(19.8 \%)$ & $41(41.4 \%)$ & $58(58.6 \%)$ & 0.908 \\
\hline R34 & ${ }^{9}$ AANSAIDS & $81(16.2 \%)$ & $29(35.8 \%)$ & $52(64.2 \%)$ & 0.425 \\
\hline R35 & Insulin & $38(7.6 \%)$ & $10(26.3 \%)$ & $28(73.7 \%)$ & 0.095 \\
\hline R36 & ${ }^{10} \mathrm{~N}-\mathrm{IDM}$ & $72(14.4 \%)$ & $22(30.6 \%)$ & $50(69.4 \%)$ & 0.087 \\
\hline R37 & MPH & $2.64 \pm 1.4$ & $2.59 \pm 1.3$ & $2.67 \pm 1.4$ & 0.570 \\
\hline R38 & TPA & $47.68 \pm 44.3$ & $45.08 \pm 41.7$ & $49.45 \pm 45.9$ & 0.280 \\
\hline R39 & IPN & $1.16 \pm 0.8$ & $1.11 \pm 0.8$ & $1.19 \pm 0.8$ & 0.240 \\
\hline
\end{tabular}

+ Significant cutoff of risk covariate is $\mathrm{p}<0.05$ (12 covariates); ${ }^{1} \mathrm{ADba}$ : Ave Dias before angio; ${ }^{2} \mathrm{ASba}$ : Avg Sys before angio; ${ }^{3}$ FHPCVD: Family Hx of Premature CVD; ${ }^{4}$ FHD: Family Hx of Diabetes; ${ }^{5} \mathrm{HCRI}$ : HMG-Co Reductase Inhibitors (Statins); ${ }^{6} \mathrm{OAA}$ : Other Antilipemic Agents (not statins); ${ }^{7} \mathrm{CCB}$ : Calcium Channel Blockers; ${ }^{8}$ AP/AC: Anti-Platelet/Anti-Coagulants; ${ }^{9}$ AANSAIDS: Anti-Anginals and NSAIDS; ${ }^{10}$ N-IDM: Non-Insulin Diabetes Medications; MPH: maximum plaque height; TPA: total plaque area; IPN: intra-plaque neovascularization, NSAIDS: non-steroidal anti-inflammatory drugs; ARB: angiotensin receptor blocker; ACE: angiotensin-converting enzyme; BMI: body mass index, T1D: Type 1 diabetes, T2D: Type 2 diabetes 


\section{Appendix B}

\section{Three Classifiers}

\section{A. Random forest}

$\mathrm{RF}$ is an ensemble ML-based learning algorithm that is comprised of various decision trees (DT). Each DT in the RF classifier receives a complete covariate set and performs the classification of the CVD risk. It follows a voting-based method, where the independent risk predictions by all DT are combined, and final risk prediction is performed using majority voting. Table 20 shows the set of tuning parameters used in the RF classifier.

B. Support Vector Machine with radial the basis function

Support Vector Machine (SVM) splits the input dataset samples into two classes that maximize the margin between two classes using optimal decision boundary. The classification framework is binary and was performed using a "one-vs-all" method. When the input dataset is non-linear (linearly inseparable), we use kernel functions such as RBF (kernel= "RBF"). When the input dataset is linearly separable, we use the linear kernel function (kernel = "linear"). We used a "RandomizedSearchCV" algorithm with RBF kernel for the optimization of tuning parameters. The three optimized tuning parameters used for the SVM classifier with the "RBF" kernel are provided in Table 21.

C. Linear discriminant analysis

Linear discriminant analysis (LDA)is a dimensionality reduction method and is a generalization of Fisher LDA. This is an ML-based paradigm and is used to find the linear combination of covariates that classify into binary class or multiclass, where each class has equal covariance matrices. The set of optimized tuning parameters required for the LDA classifier are provided in Table 22.

\section{Appendix C}

\section{Performance evaluation metrics}

The performance of all three ML-based classifiers (LDA, SVMrbf, and RF) and all three CCVRC calculators (FRS, SCORE, and ASCVD) [23, 47] was assessed using various metrics such as sensitivity, specificity, false-positive rate (FPR), false-negative rate (FNR), positive predictive value (PPV), negative predictive value (NPV), accuracy and areaunder-the-curve (AUC). The AUC score for all the matrices
Table 20 Optimized tuning parameters of the RF algorithm

\begin{tabular}{lll}
\hline SN & Tuning parameter & Optimized value \\
\hline 1 & Total number of trees & 617 \\
2 & Maximum depth & 142 \\
2 & Minimum sample split & 7 \\
3 & Minimum sample leaf & 4 \\
4 & Criterion & Gini \\
\hline
\end{tabular}

Table 21 Three optimized tuning parameters of the SVM algorithm

\begin{tabular}{lll}
\hline SN & Tuning parameters & $\begin{array}{l}\text { opti- } \\
\text { mized } \\
\text { value }\end{array}$ \\
\hline 1 & Kernel & $\begin{array}{c}\text { Radial } \\
\text { basis } \\
\text { func- } \\
\text { tion } \\
\text { (RBF) }\end{array}$ \\
& & 2 \\
3 & C & 0.1 \\
\hline
\end{tabular}

Table 22 Three optimized tuning parameters of the LDA algorithm

\begin{tabular}{lll}
\hline SN & Tuning Parameters & Optimized Value \\
\hline 1 & Solver & Singular value \\
& & decomposition \\
2 & Tol & $1.0 \mathrm{e}-4$ \\
3 & Store Covariance & False \\
\hline
\end{tabular}

was computed using a $2 \mathrm{X} 2$ confusion matrix that contains false-positive (FP), false negative (FN), true-positive (TP), and true-negative (TN). The $2 \mathrm{X} 2$ confusion matrix compares the two categorical covariates - ground-truth labels and the predicted risk labels from the ML-based classifiers. The true-positive (TP) shows the total number of times the classifier has correctly recognized the positive class and the true-negative (TN) shows the total number of times the classifier has correctly recognized the negative class. The false-positive defines the total number of times the classifier has correctly allocated a positive label to the patient with respect to the actual negative ground-truth label. Similarly, the false-negative defines the total number of times the classifier has correctly allocated a negative label to the patient with respect to the actual positive ground-truth label. Since we have binary-class risk labels, we first compute confusion 
Table 23 Mathematical expressions for performance evaluation metrics

\begin{tabular}{|c|c|c|c|c|}
\hline $\mathrm{SN}$ & Performance measure & Mathematical ex & pressiol & \\
\hline 1 & Sensitivity, $\%$ & Sensitivity $=$ & $\left.\frac{\mathrm{TP}}{(\mathrm{TP}+\mathrm{FN})}\right]$ & $\times 100$ \\
\hline 2 & Specificity, \% & Specif icity $=$ & $\left.\frac{\mathrm{TN}}{(\mathrm{TN}+\mathrm{FP})}\right]$ & $\times 100$ \\
\hline 3 & False Predictive Rate (FPR), $\%$ & $\mathrm{FPR}=$ & $\left.\frac{\mathrm{FP}}{(\mathrm{FP}+\mathrm{TN})}\right]$ & $\times 100$ \\
\hline 4 & False Negative Rate (FNR), $\%$ & $\mathrm{FNR}=[$ & $\left.\frac{\mathrm{FN}}{(\mathrm{FN}+\mathrm{TP})}\right]$ & $\times 100$ \\
\hline 5 & $\begin{array}{l}\text { Positive Predictive Value } \\
\text { (PPV), \% }\end{array}$ & $\mathrm{PPV}=$ & $\left.\frac{\mathrm{TP}}{(\mathrm{TP}+\mathrm{FP})}\right]$ & $\times 100$ \\
\hline 6 & $\begin{array}{l}\text { Negative Predictive Value } \\
\text { (NPV), \% }\end{array}$ & $\mathrm{NPV}=$ & $\left.\frac{\mathrm{TN}}{(\mathrm{TN}+\mathrm{FN})}\right]$ & $\times 100$ \\
\hline 7 & Mean Accuracy (ACC), \% & $\mathrm{ACC}=\left[\frac{\mathrm{TP}}{(\mathrm{TP}+\mathrm{FP}-}\right.$ & $\left.\frac{-\mathrm{TN}}{\mathrm{TN}+\mathrm{FN})}\right]$ & $\times 100$ \\
\hline
\end{tabular}

matrices and the corresponding four sets of TP, TN, FP and $\mathrm{FN}$, then we use these four sets to find the performance matrices involving sensitivity, specificity, FPR, FNP, PPV, NPV, and accuracy (Table 23).

In this study, we also calculate the receiver operating characteristics (ROC) curve. It is a plot between the truepositive rate and the false-positive rate. In other word, it is a plot between sensitivity and 100-specificity of the probability predictions of the ML classifiers at various cut-off points. Every point on the ROC curve indicates the sensitivity/specificity pair at the cut-off point. The upper left corner of the plot shows $100 \%$ sensitivity and specificity.

Supplementary Information The online version contains supplementary material available at https://doi.org/10.1007/s00296-021-05062-4.

\section{References}

1. Cross $M$ et al (2014) The global burden of rheumatoid arthritis: estimates from the global burden of disease 2010 study. Ann Rheum Dis 73(7):1316-1322

2. van Vollenhoven RF (2009) Sex differences in rheumatoid arthritis: more than meets the eye. BMC Med 7(1):12

3. Crowson CS et al (2013) Rheumatoid arthritis and cardiovascular disease. Am Heart J 166(4):622-628

4. Domingues VDS et al (2021) Increased short-term risk of cardiovascular events in inflammatory rheumatic diseases: results from a population-based cohort. Rheumatol Int 41(2):311-318

5. Arts E, et al. (2014) Performance of four current risk algorithms in predicting cardiovascular events in patients with early rheumatoid arthritis. Anne Rheum Dis 2014: annrheumdis-2013-204024

6. Crowson CS et al (2012) Usefulness of risk scores to estimate the risk of cardiovascular disease in patients with rheumatoid arthritis. Am J Cardiol 110(3):420-424

7. Arts E, et al. (2015) Prediction of cardiovascular risk in rheumatoid arthritis: performance of original and adapted SCORE algorithms. Ann Rheum Dis 2015: annrheumdis-2014-206879
8. Escalante A, Haas RW, Del Rincón I (2005) Paradoxical effect of body mass index on survival in rheumatoid arthritis: role of comorbidity and systemic inflammation. Arch Intern Med 165(14):1624-1629

9. Urruela MA, Suarez-Almazor ME (2012) Lipid paradox in rheumatoid arthritis: changes with rheumatoid arthritis therapies. Curr Rheumatol Rep 14(5):428-437

10. Gabriel SE (2010) Heart disease and rheumatoid arthritis: understanding the risks. Ann Rheum Dis 69(1):61-64

11. del Rincón ID et al (2001) High incidence of cardiovascular events in a rheumatoid arthritis cohort not explained by traditional cardiac risk factors. Arthritis Rheum 44(12):2737-2745

12. Jamthikar AD, et al. (2021) Multiclass machine learning vs. conventional calculators for stroke/CVD risk assessment using carotid plaque predictors with coronary angiography scores as gold standard: a 500 participants study. Int J Cardiovasc Imag 37(4): 1171-1187

13. Araki T et al (2016) A new method for IVUS-based coronary artery disease risk stratification: a link between coronary and carotid ultrasound plaque burdens. Comput Methods Programs Biomed 124:161-179

14. Biswas $\mathrm{M}$ et al (2021) A review on joint carotid intima-media thickness and plaque area measurement in ultrasound for cardiovascular/stroke risk monitoring: artificial intelligence framework. J Digit Imaging 34(3):581-604

15. Cuadrado-Godia E et al (2018) Morphologic TPA (mTPA) and composite risk score for moderate carotid atherosclerotic plaque is strongly associated with $\mathrm{HbA} 1 \mathrm{c}$ in diabetes cohort. Comput Biol Med 101:128-145

16. Jamthikar A et al (2019) A low-cost machine learning-based cardiovascular/stroke risk assessment system: integration of conventional factors with image phenotypes. Cardiovasc Diagn Ther 9(5):420-430

17. Jamthikar AD et al (2020) Cardiovascular risk assessment in patients with rheumatoid arthritis using carotid ultrasound B-mode imaging. Rheumatol Int 40(12):1921-1939

18. Khanna NN et al (2019) Effect of carotid image-based phenotypes on cardiovascular risk calculator: AECRS10. Med Biol Eng Comput 57(7):1553-1566

19. Puvvula A et al (2020) Morphological carotid plaque area is associated with glomerular filtration rate: a study of south asian indian patients with diabetes and chronic kidney disease. Angiology 71(6):520-535

20. Viswanathan V et al (2020) Does the carotid bulb offer a better 10-Year CVD/stroke risk assessment compared to the common carotid artery? A 1516 ultrasound scan study. Angiology 71(10):920-933

21. del Rincón I et al (2005) Relative contribution of cardiovascular risk factors and rheumatoid arthritis clinical manifestations to atherosclerosis. Arthritis Rheum 52(11):3413-3423

22. del Rincón I et al (2015) Systemic inflammation and cardiovascular risk factors predict rapid progression of atherosclerosis in rheumatoid arthritis. Ann Rheum Dis 74(6):1118-1123

23. Jamthikar A et al (2020) Ultrasound-based stroke/cardiovascular risk stratification using framingham risk score and ASCVD Risk Score based on "integrated vascular age" instead of "chronological age": a multi-ethnic study of Asian Indian, Caucasian, and Japanese cohorts. Cardiovasc Diagn Ther 10(4):939-954

24. Saba L et al (2019) Global perspective on carotid intima-media thickness and plaque: should the current measurement guidelines be revisited? Int Angiol 38(6):451-465

25. Soriano-Valdez D et al (2021) The basics of data, big data, and machine learning in clinical practice. Clin Rheumatol 40:11-23

26. Porcu $\mathrm{M}$ et al (2020) Carotid plaque imaging profiling in subjects with risk factors (diabetes and hypertension). Cardiov Diagn Thera 10(4):1005 
27. Ikeda $\mathrm{N}$ et al (2015) Improved correlation between carotid and coronary atherosclerosis SYNTAX score using automated ultrasound carotid bulb plaque IMT measurement. Ultrasound Med Biol 41(5):1247-1262

28. Mach F et al (2019) 2019 ESC/EAS Guidelines for the management of dyslipidaemias: lipid modification to reduce cardiovascular risk: the task force for the management of dyslipidaemias of the european society of cardiology (ESC) and european atherosclerosis society (EAS). Eur Heart J 41(1):111-188

29. Knuuti J et al (2019) 2019 ESC Guidelines for the diagnosis and management of chronic coronary syndromes: the task force for the diagnosis and management of chronic coronary syndromes of the european society of cardiology (ESC). Eur Heart J 41(3):407-477

30. Johri AM et al (2016) Focused vascular ultrasound for the assessment of atherosclerosis: a proof-of-concept study. J Am Soc Echocardiogr 29(9):842-849

31. Johri AM et al (2013) Can carotid bulb plaque assessment rule out significant coronary artery disease? A comparison of plaque quantification by two-and three-dimensional ultrasound. J Am Soc Echocardiogr 26(1):86-95

32. Stein JH et al (2008) Use of carotid ultrasound to identify subclinical vascular disease and evaluate cardiovascular disease risk: a consensus statement from the American society of echocardiography carotid intima-media thickness task force. Endorsed by the society for vascular medicine. J Am Soc Echocardiogr 21(2):93-111

33. Molinari F et al (2012) Automated carotid IMT measurement and its validation in low contrast ultrasound database of 885 patient Indian population epidemiological study: results of AtheroEdge ${ }^{\mathrm{TM}}$ Software. Int Angiol 31(1):42-53

34. Molinari F et al (2012) Completely automated multiresolution edge snapper-a new technique for an accurate carotid ultrasound IMT measurement: clinical validation and benchmarking on a multi-institutional database. IEEE Trans Image Process 21(3):1211-1222

35. Molinari F, Zeng G, Suri JS (2010) Intima-media thickness: setting a standard for a completely automated method of ultrasound measurement. IEEE Trans Ultrason Ferroelectr Freq Control 57(5):1112-1124

36. Saba L et al (2012) Intima media thickness variability (IMTV) and its association with cerebrovascular events: a novel marker of carotid therosclerosis? Cardiovasc Diagn Ther 2(1):10-18

37. Hao M, Wang Y, Bryant SH (2014) An efficient algorithm coupled with synthetic minority over-sampling technique to classify imbalanced PubChem BioAssay data. Anal Chim Acta 806:117-127

38. Jamthikar AD et al (2020) Cardiovascular risk assessment in patients with rheumatoid arthritis using carotid ultrasound B-mode imaging. Rheumatol Internat 2020:1-19

39. Jamthikar A et al (2019) A special report on changing trends in preventive stroke/cardiovascular risk assessment Via B-mode ultrasonography. Curr Atheroscler Rep 21(7):25

40. Saba L et al (2017) Plaque tissue morphology-based stroke risk stratification using carotid ultrasound: a polling-based PCA learning paradigm. J Med Syst 41(6):98

41. Jónsson H, G Cherubini G, Eleftheriou E (2020) Convergence behavior of DNNs with mutual-information-based regularization. Entropy (Basel) 22(7).

42. Jamthikar A et al (2020) Cardiovascular/stroke risk prevention: a new machine learning framework integrating carotid ultrasound image-based phenotypes and its harmonics with conventional risk factors. Indian Heart J 72(4):258-264

43. Acharya UR et al (2013) Understanding symptomatology of atherosclerotic plaque by image-based tissue characterization. Comput Methods Programs Biomed 110(1):66-75

44. Araki T et al (2016) PCA-based polling strategy in machine learning framework for coronary artery disease risk assessment in intravascular ultrasound: a link between carotid and coronary grayscale plaque morphology. Comput Methods Programs Biomed 128:137-158

45. Maniruzzaman M et al (2019) Statistical characterization and classification of colon microarray gene expression data using multiple machine learning paradigms. Comput Methods Programs Biomed 176:173-193

46. Maniruzzaman M et al (2017) Comparative approaches for classification of diabetes mellitus data: machine learning paradigm. Comput Methods Programs Biomed 152:23-34

47. Jamthikar A et al (2020) Cardiovascular/stroke risk predictive calculators: a comparison between statistical and machine learning models. Cardiovasc Diagn Ther 10(4):919-938

48. Fariniuk LF et al (2017) Efficacy of protaper instruments during endodontic retreatment. Indian J Dent Res 28(4):400-405

49. Fernandes Filho J et al (2017) Evaluation and comparison of five skinfold calipers. Nutr Hosp 34(1):111-115

50. Banchhor SK, et al. (2017) Relationship between automated coronary calcium volumes and a set of manual coronary lumen volume, vessel volume and atheroma volume in japanese diabetic cohort. J Clin Diagn Res 11(6): Tc9-Tc14

51. Saba L et al (2016) Inter-observer variability analysis of automatic lung delineation in normal and disease patients. J Med Syst 40(6): 142

52. Johri AM, et al. (2021) Role of artificial intelligence in cardiovascular risk prediction and outcomes: comparison of machinelearning and conventional statistical approaches for the analysis of carotid ultrasound features and intra-plaque neovascularization. Int J Cardiovasc Imaging.

53. Abraham A et al (2014) Machine learning for neuroimaging with scikit-learn. Front Neuroinform 8:14

54. Cruz GV et al (2021) Machine learning reveals the most important psychological and social variables predicting the differential diagnosis of rheumatic and musculoskeletal diseases. Rheumatol Internat 2021:1-10

55. Kataria S, Ravindran V (2018) Digital health: a new dimension in rheumatology patient care. Rheumatol Int 38(11):1949-1957

56. Schmajuk G, Yazdany J (2017) Leveraging the electronic health record to improve quality and safety in rheumatology. Rheumatol Int 37(10): 1603-1610

57. Khanna NN et al (2019) Rheumatoid arthritis: atherosclerosis imaging and cardiovascular risk assessment using machine and deep learning-based tissue characterization. Curr Atheroscler Rep 21(2):7

58. Jamthikar A et al (2019) A low-cost machine learning-based cardiovascular/stroke risk assessment system: integration of conventional factors with image phenotypes. Cardiovasc Diagn Therapy 9(5):420

59. Jamthikar A, et al. (2020) Cardiovascular/stroke risk prevention: a new machine learning framework integrating carotid ultrasound image-based phenotypes and its harmonics with conventional risk factors. Indian Heart J

60. Jamthikar A et al (2020) Cardiovascular/stroke risk predictive calculators: a comparison between statistical and machine learning models. Cardiovasc Diagn Therapy 10(4):919-938

61. Gastounioti A et al (2015) A novel computerized tool to stratify risk in carotid atherosclerosis using kinematic features of the arterial wall. IEEE J Biomed Health Inform 19(3):1137-1145

62. Unnikrishnan P, et al. (2016) Development of health parameter model for risk prediction of CVD using SVM. Comput Mathemat Methods Med 2016

63. Weng SF et al (2017) Can machine-learning improve cardiovascular risk prediction using routine clinical data? PLoS ONE 12(4):e0174944 
64. Ambale-Venkatesh B, et al. (2017) Cardiovascular event prediction by machine learning: the Multi-Ethnic Study of Atherosclerosis. Circulation Res, Circresaha. 117.311312

65. Araki $\mathrm{T}$ et al (2017) Stroke risk stratification and its validation using ultrasonic echolucent carotid wall plaque morphology: a machine learning paradigm. Comput Biol Med 80:77-96

66. Nakamura M et al (2013) LVQ-SMOTE - learning vector quantization based synthetic minority over-sampling technique for biomedical data. BioData Min 6(1):16

67. Saba L et al (2021) Ultrasound-based internal carotid artery plaque characterization using deep learning paradigm on a supercomputer: a cardiovascular disease/stroke risk assessment system. Int J Cardiovasc Imaging 37(5):1511-1528

68. Skandha SS et al (2020) 3-D optimized classification and characterization artificial intelligence paradigm for cardiovascular/ stroke risk stratification using carotid ultrasound-based delineated plaque: Atheromatic ${ }^{\mathrm{TM}}$ 20. Comput Biol Med 125:103958

69. Banchhor SK et al (2017) Wall-based measurement features provides an improved IVUS coronary artery risk assessment when fused with plaque texture-based features during machine learning paradigm. Comput Biol Med 91:198-212

70. Kakadiaris IA et al (2018) Machine learning outperforms ACC/AHA CVD risk calculator in MESA. J Am Heart Assoc 7(22): 009476

Publisher's Note Springer Nature remains neutral with regard to jurisdictional claims in published maps and institutional affiliations.

\section{Authors and Affiliations}

\section{George Konstantonis $^{1} \cdot$ Krishna V. Singh $^{2}$ (D) . Petros P. Sfikakis ${ }^{1}$ (D) . Ankush D. Jamthikar ${ }^{3,4}$ (D) George D. Kitas ${ }^{5,6}$ (D) Suneet K. Gupta ${ }^{7}$ - Luca Saba ${ }^{8}$ (D) Kleio Verrou ${ }^{9} \cdot$ Narendra N. Khanna $^{10}$ (D) Zoltan Ruzsa ${ }^{11}$ (D) Aditya M. Sharma ${ }^{12}$ - John R. Laird ${ }^{13}$ (D) Amer M. Johri ${ }^{14}$ (D) Manudeep Kalra ${ }^{15}$ (D) Athanasios Protogerou $^{16}$. Jasjit S. Suri ${ }^{17}$}

1 Rheumatology Unit, National Kapodistrian University of Athens, Athens, Greece

2 Research Intern, AtheroPoint ${ }^{\mathrm{TM}}$, Roseville, CA, USA

3 Research Scientist, AtheroPoint ${ }^{\mathrm{TM}}$, USA, Roseville, CA, USA

4 Visvesvaraya National Institute of Technology, Nagpur, India

5 Academic Affairs, Dudley Group NHS Foundation Trust, Dudley, UK

6 Arthritis Research UK Epidemiology Unit, Manchester University, Manchester M13, UK

7 Department of Computer Science, Bennett University, Gr. Noida, India

8 Department of Radiology, University of Cagliari, Cagliari, Italy

9 Department of Medicine, National and Kapodistrian University of Athens, Athens, Greece
10 Department of Cardiology, Indraprastha Apollo Hospitals, New Delhi, India

11 Department of Internal Medicines, Invasive Cardiology Division, University of Szeged, Szeged, Hungary

12 Division of Cardiovascular Medicine, University of Virginia, Charlottesville, VA, USA

13 Heart and Vascular Institute, Adventist Health St. Helena, St Helena, CA, USA

14 Department of Medicine, Division of Cardiology, Queen's University, Kingston, ON, Canada

15 Department of Radiology, Massachusetts General Hospital, 55 Fruit Street, Boston, MA, USA

16 Cardiovascular Prevention Unit, Department of Pathophysiology, National Kapodistrian University of Athens, Athens, Greece

17 Stroke Monitoring and Diagnostic Division, AtheroPoint ${ }^{\mathrm{TM}}$, Roseville, CA 95661, USA 